\title{
An innovative framework for probabilistic-based structural assessment with an application to existing reinforced concrete structures
}

\author{
José C. Matos ${ }^{1}$, Paulo J. S. Cruz ${ }^{2}$, Isabel B. Valente ${ }^{3}$, Luís C. Neves ${ }^{4}$, Vicente N. Moreira ${ }^{5}$ \\ ISISE, Department of Civil Engineering, University of Minho, Guimarães, Portugal
}

\footnotetext{
${ }^{1}$ Assistant Professor, ISISE, Department of Civil Engineering, University of Minho, Campos de Azurém, 4800058 Guimarães, Portugal. Phone: +351 253510 200, E-mail: jmatos@ civil.uminho.pt (corresponding author)

${ }^{2}$ Full Professor, Lab2PT, School of Architecture, University of Minho, Campos de Azurém, 4800-058 Guimarães, Portugal. Phone: +351 253510 505, E-mail: pcruz@arquitectura.uminho.pt

${ }^{3}$ Assistant Professor, ISISE, Department of Civil Engineering, University of Minho, Campos de Azurém, 4800058 Guimarães, Portugal. Phone: +351 253510 200, E-mail: isabelv@ civil.uminho.pt

${ }^{4}$ Lecturer, Transportation Engineering Centre (NTEC), University of Nottingham, NTEC Building, NG7 2RD, UK, Phone: +44 011584 67365, E-mail: luis.neves@nottingham.ac.uk

${ }^{5} \mathrm{PhD}$ candidate, ISISE, Department of Civil Engineering, University of Minho, Campos de Azurém, 4800-058 Guimarães, Portugal. Phone: +351 253510 200, E-mail: vicente.nmoreira@gmail.com
} 


\section{ABSTRACT}

A novel framework for probabilistic-based structural assessment of existing structures, which combines model identification and reliability assessment procedures, considering in an objective way different sources of uncertainty, is presented in this paper. A short description of structural assessment applications, provided in literature, is initially given. Then, the developed model identification procedure, supported in a robust optimization algorithm, is presented. Special attention is given to both experimental and numerical errors, to be considered in this algorithm convergence criterion. An updated numerical model is obtained from this process. The reliability assessment procedure, which considers a probabilistic model for the structure in analysis, is then introduced, incorporating the results of the model identification procedure. The developed model is then updated, as new data is acquired, through a Bayesian inference algorithm, explicitly addressing statistical uncertainty. Finally, the developed framework is validated with a set of reinforced concrete beams, which were loaded up to failure in laboratory.

Keywords: Structural assessment, Uncertainty sources, Model identification, Optimization algorithm, Reliability assessment, Bayesian inference, Reinforced concrete structures 


\section{Introduction}

Structural assessment comprises all activities required to evaluate the condition of structures for future use, in particular, regarding safety. During structural assessment of existing structures a wide range of sources of uncertainty can be identified. In order to consider them, reliability algorithms are commonly used. Structural safety is quantified through the reliability index, or the probability of failure, obtained from the comparison of resistance and effect of loads probability density functions (PDFs) [1-3].

Several authors have used probabilistic-based safety assessment procedures to assess existing structures, having shown that the conclusions can be dramatically different from those obtained using existing codes [4-10]. More recently, Bayesian inference was introduced to improve the quality of probabilistic models for both resistance and effect of loads, using data collected from the structure under analysis $[11,12]$.

The use of nonlinear finite element analysis (NL FEA) methods in structural assessment procedures, although computational costly, enables a more realistic estimation of the structural response, both in service and ultimate limit states. Bergmeister et al. [13] introduced a probabilistic-based safety assessment concept for reinforced concrete structures that integrates NL FEA software with reliability-based algorithms.

For existing structures, the available information regarding used materials (e.g. class of concrete or steel) and geometry is always scarce. Moreover, the retrieval of samples for laboratory tests is often restricted. As a result, the applicability of Bayesian updating directly considering material and geometric properties has limited applicability. Therefore, some authors used model identification techniques to estimate structural parameters based on performance measures. A review of these procedures is provided in [14]. Accordingly, Novák et al. [15] developed a complex methodology for structural assessment of existing structures, which combines structural analysis and reliability algorithms with new modules for model identification.

In this paper, a novel framework for probabilistic-based structural assessment of existing structures is presented. This framework combines some of structural assessment techniques above outlined with a new methodology to identify optimal solutions, based on an evolutionary algorithm and a hybrid decision-making procedure, and a Bayesian inference tool, providing the objective treatment of uncertainties. In the first step, model parameters, in particular material (e.g. steel yield stress, concrete strength), geometric and mechanic properties are estimated considering a minimization procedure between observed performance and performance predicted using a non- 
linear finite element model (NL FEM). A convergence criterion is defined considering the expected accuracy of experimental and numerical data. The minimization procedure yields a set of near optimal solutions, from which the best model is selected considering the probability of each solution occurring based on previous knowledge. If deemed adequate, an expert judgment can be employed within this selection procedure. Once selected, the deterministic model is converted into a probabilistic model by considering randomness in model parameters, through the adoption of appropriate PDFs. Bayesian inference is then used to update each model parameter with new acquired data from material and geometric properties. This way, the statistical uncertainty is explicitly considered. Structural safety is respectively assessed in a continuous basis through this framework.

This framework can be classified, according to SAMCO report [16], as a level 5 assessment class (model-based assessment of existing structures), once it combines probabilistic simulation methods, with a stochastic NL FEM and data from testing and measurement of material properties and dimensions. Although this methodology can be applied to new structures, its application aims at better characterizing existing structures for which limited information exists. The effectiveness of both model identification and reliability assessment procedure, with an integrated Bayesian inference approach, is supported in the reliability of such data. Accordingly, the developed framework, which addresses different sources of uncertainties, is tested and validated with a set of reinforced concrete beams, which were loaded up to failure in laboratory. This controlled experiment is crucial since, unlike real structures, destructive tests can be extensively employed to evaluate the accuracy of the prediction.

\section{Probabilistic-based structural assessment}

The proposed probabilistic-based structural assessment methodology can be divided in two main steps, Figure 1. In the first step a deterministic analysis is used to estimate the most important model parameters, based on the combination of numerical methods and experimental data. This procedure, denoted as model identification, searches for expected values of material, geometric and mechanic structural properties. This data is then used to define the probabilistic distributions of structural parameters, used in the reliability assessment of the structure.

The main objective of model identification procedure is to obtain the most likely values of model parameters, consistent with observed structural performance. Within this procedure, numerical results are fitted to collected data from real structure, by adjusting model parameter values. This procedure is accomplished by using an optimization algorithm, with the objective of minimizing the difference between obtained numerical results and measured data, expressed by a fitness function. The optimization procedure stops when the improvement in this 
function is equal or lower than a threshold value. The main result of this procedure is a structural model that can, with acceptable accuracy, predict the structure performance.

The aim of reliability assessment of the structure is to evaluate its condition for future use, considering randomness in model parameters. In order to do that, a prior distribution is assigned to each model parameter. This distribution may be then updated through a Bayesian inference procedure, with complementary data obtained by visual inspection, non-destructive tests or permanent monitoring systems. A posterior distribution is respectively computed, being obtained an updated performance indicator for the structure under evaluation.

The main drawback of this methodology is its computational cost. In order to surpass this, an initial sensitivity analysis is recommended. The main objective of this analysis is to identify the parameters with a higher impact on the overall structural behavior. This analysis consists in evaluating the fitness function variation with each input parameter. An importance measure, $b_{k}$, is obtained for each parameter, expression (1),

$b_{k}=\sum_{i=1}^{n}\left(\Delta y_{k} / y_{m}\right) /\left(\Delta x_{k} / x_{m}\right) \cdot C V[\%]$

with $\Delta y_{k}$ the variation in structural response due to a deviation of $\Delta x_{k}$ in input parameter mean value $x_{m}, y_{m}$ the average response, $n$ the number of generated parameters and CV the parameter coefficient of variation.

\subsection{Model identification}

In a first step, and according to Figure 1, model identification is performed to obtain an updated deterministic numerical model. During this procedure, model parameters are obtained from an automatic adjustment process to measured data (Figure 2). In the model identification procedure, unknown variables were taken as uncorrelated.

For each set of values of the different parameters (e.g. steel yield stress, concrete compressive strength), a NL FEM is defined and the obtained results $\left(y_{i}^{\text {num }}\right)$ are compared with the corresponding experimental results $\left(y_{i}^{\exp }\right.$

), Figure $3 a$, through the fitness function, $f$, given by equation (2),

$f=\sum_{i=1}^{n}\left|y_{i}^{\text {num }}-y_{i}^{\text {exp }}\right| / \max \left(y^{\text {exp }}\right) \cdot 1 / n[\%]$ 
where $n$ is the number of evaluated points from the real structure. It is important to point out that this function is normalized and so, it can be used with different transducers, measuring different parameters, placed in different sections of the structure in evaluation.

The adjustment procedure consists in minimizing the distance between measured and numerical data, given by fitness function (2). A wide range of optimization numerical methods can be used to solve this problem. On one hand, gradient based methods are usually less computationally expensive, but less robust, frequently converging to local minima. On the other hand, non-gradient methods, in particular, population-based meta-heuristic methods tend to be significantly more robust, increasing the probability of finding global minima and avoiding the need to compute gradients. However, they are usually very computationally demanding. Herein, a population-based method, described in [17] and denoted as evolutionary strategies - plus version, was used. To further enhance the robustness of the method, the algorithm was run multiple times, using different initial randomly generated populations to avoid falling into local minima.

According to Figure 2, the fitness function convergence criterion, given by equation (3), may be used as an optimization algorithm stopping criterion,

$\Delta f=\left|f_{i+n}-f_{i}\right| \leq \varepsilon$

with $f$ the minimum fitness function value from a population of models for generation $i$ and $i+n$, and $n$ the defined gap between these two generations. If the difference between these two values is less than or equal to a pre-specified threshold value, $\varepsilon$, the algorithm stops, and all solutions repeating the criterion considered as potential optimum. If this difference is higher than the threshold, then the algorithm continues for further iterations.

In order to limit the probability of over fitting, optimization is conducted, not to find the best solution, but a group of solutions associated with a fitness under a given threshold. In fact, when using such a model identification procedure, two sources of errors must be considered: experimental and numerical errors [18-21]. Consequently, it is assumed that when computing the difference between numerical and experimental data, according to fitness function (2), results associated with a fitness below the expected amplitude of errors are considered as optimal, Figure $3 \mathrm{~b}$. The threshold value, $\varepsilon$, is then computed through the law of propagation of uncertainty [22], combining both measurement and modeling errors [14]. 
A population of models that respect the convergence criterion is selected through this procedure. These models are then analyzed by a hybrid process, based in the probability of occurrence of each model and, potentially, expert judgment is used to select the most likely result. The most likely model (i.e., the model with highest probability of occurrence) is used in the following steps.

\subsubsection{Errors}

Errors play a major role in model identification. They may arise from many sources, being the most significant the measurement and modeling errors. Model identification is always limited by the combination of these two sources of errors [18-21]. Accordingly, numerical models are updated until a certain limit (threshold value), obtained through the contribution of these two components, is attained.

Measurement error, $u_{\text {exp }}$, corresponds to the difference between real and measured quantities in a single measurement. It usually results from equipment and on-site installation limitations. Accordingly, different sources may be defined for this type of error [23-25]: (1) sensor accuracy, usually reported by the manufacturer; (2) stability (e.g. ambient vibrations); (3) robustness (e.g. environmental effects due to temperature); (4) load positioning; and (5) load intensity.

Modeling error, $u_{\text {num }}$, corresponds to the difference between the response of a given model and that of an ideal model which accurately represents the structural behavior. It is possible to divide this error in three main components, Figure $4[18,19,26]$ : (a) $u_{1}$, discrepancy between the behavior of a mathematical model and that from the real structure; (b) $u_{2}$, numerical error in solving the partial differential equations (e.g. finite element method, mesh discretization); and (c) $u_{3}$, inaccurate assumptions made during simulation (e.g. boundary condition such as support characteristics, applied load steps). Component $u_{1}$ is extremely difficult to quantify, as it is problem dependent and it can be minimized through modeling expertise. Ravindram et al. [19] proposes to consider this component as null when an ideal situation is assumed.

The component errors $u_{2}$ and $u_{3}$ can be computed by comparing obtained results from numerical model, used in model identification procedure, with a reference model which presents a more refined mesh and a higher number of load steps [20]. This comparison is established through equation (4), 
$u=\sum_{i=1}^{n}\left|y_{i}^{\text {num } 1}-y_{i}^{\text {num } 0}\right| / \max \left(y^{\text {num } 0}\right) \cdot 1 / n[\%]$

where $y^{\text {num } 0}$ is the reference model value, $y^{\text {numl }}$ is the obtained value from numerical model, used in model identification, and $n$ the number of comparing points.

\subsubsection{Convergence criterion}

Different fitness function convergence criteria can be used in model identification. Robert-Nicoud et al. [18] proposes a procedure which considers as candidate models those that present a fitness function value lower than a pre-specified threshold. Considering $y$ as the real value of a measurable quantity, $y^{\exp }$ the measured value and $y^{\text {num }}$ the numerical value (Figure $3 \mathrm{~b}$ ), the following relationship can be defined, expression (5),

$y=y^{\text {exp }}+u_{\text {exp }}=y^{\text {num }}+u_{\text {num }}=y^{\text {num }}+\left(u_{1}+u_{2}+u_{3}\right)$

Model identification procedure aims to minimize the absolute value of the difference between $y^{\text {num }}$ and $y^{\text {exp }}$, known as residual, $q$. Considering expression (5), and considering that both $u_{\text {num }}$ and $u_{\text {exp }}$ might be positive or negative, the residual is given by equation (6),

$q=\left|y^{\text {num }}-y^{\text {exp }}\right| \Leftrightarrow q \leq\left|y^{\text {num }}\right|+\left|y^{\text {exp }}\right| \leq|u| \leq\left|u_{\text {num }}\right|+\left|u_{\text {exp }}\right| \leq\left|u_{1}\right|+\left|u_{2}\right|+\left|u_{3}\right|+\left|u_{\text {exp }}\right|$

The proposed convergence criterion by Robert-Nicoud et al. [18] may be reduced to expression (7),

$f(q) \leq u$

where $f(q)$ is the computed residual and $u$ the global uncertainty.

The convergence criterion, given by equation (3), defines that the improvement in fitness function value, $\Delta f$, from two models separated of a pre-specified gap, $n$, should be lower than or equal to a threshold value, $\varepsilon$. Accordingly, considering the methodology proposed by Robert-Nicoud et al. [18], the convergence criterion may be reduced to expression (8),

$\Delta f=\left|f_{i+n}-f_{i}\right| \Leftrightarrow \Delta f=\left|f_{i+n}\right|+\left|f_{i}\right| \leq\left|u_{i+n}\right|+\left|u_{i}\right| \leq \varepsilon$

with $f_{i}$ and $f_{i+n}$, and $u_{i}$ and $u_{i+n}$, respectively, the fitness function value and the global uncertainty, computed for generation $i$ and $i+n$. 
In order to compute the global uncertainty, $u$, it is necessary to combine both measurement and modeling errors through the law of propagation of uncertainty [22]. If errors are assumed independent, the global uncertainty is given by expression (9),

$u=\sqrt{\sum_{i=1}^{n}\left(\partial f / \partial x_{i}\right)^{2} \cdot u\left(x_{i}\right)^{2}}$

where $u\left(x_{i}\right)$ is the uncertainty associated with error source, $x_{i}$, and $\partial f / \partial x_{i}$ the partial derivative of the fitness function in order to each error source, $x_{i}$. The partial derivative evaluates the sensitivity of the fitness function with relation to each source of error, and higher values represent a strong contribution in the global uncertainty.

The fitness function, given by expression (2), is composed by two terms, respectively, a numerical and an experimental. The partial derivative, in relation to each term, can be obtained as $\partial f / \partial y^{\text {num }}=\partial f / \partial y^{\text {exp }}=1 / \max \left(y^{\text {exp }}\right)$. In order to determine the global uncertainty value, it will be necessary to separately compute the measurement and modeling error.

According to expression (8), the threshold value, $\varepsilon$, is computed by taking into consideration the sum of global uncertainties from two generations, $u_{i}$ and $u_{i+n}$, as a superior limit. These uncertainties are computed through expression (9). In this situation, the partial derivatives $\partial \Delta f / \partial u_{i+n}$ and $\partial \Delta f / \partial u_{i}$ are unitary. Once the threshold value is computed, it is used for: (i) defining potential solutions; and (ii) establish the optimization algorithm convergence criteria.

\subsubsection{Selecting of optimal solution}

Global optimization algorithms, such as evolutionary strategies [17], result in a population of optimal results. Due to the random nature of these algorithms, it is common to run the same algorithm several times, with randomly generated starting points, as to limit the probability of underperforming results and avoid falling into local minima.

From this near optimal population, the best result is taken as that with higher probability of occurrence, eventually followed by an expert judgment. Such algorithm is based on the assumption that material, geometric and mechanic properties tend to be close to the initial estimation, unless some exceptional situation is detected.

\subsection{Reliability assessment}


In a second step, and according to Figure 1, the reliability of the structure is assessed, in order to evaluate, from a probabilistic point of view, its safety level. The updated numerical model from model identification procedure, is converted into a probabilistic model by introducing randomness in its model parameters.

The probabilistic models for the properties of most structural materials, including concrete and steel, and for geometry of common elements (e.g. section dimensions and concrete cover) can be found in the literature [3, 4, 27]. The correlation between these parameters is established by proper coefficients, also given in bibliography $[3,4,27]$.

A sampling procedure is thus incorporated to randomly generate the model parameter values [1, 2]. Accordingly, an open source Latin Hypercube Sampling (LHS) [28-30] toolbox is used in the present work. There are some algorithms, such as the well-known Iman and Conover [31] or the more advanced Simulated Annealing [32] that might be used in sampling to consider the correlation between parameters. In this case, Iman and Conover algorithm was used since it was already incorporated in the LHS toolbox.

Then, for each set of generated values, the updated numerical model is analyzed with a NL FEA software, being the obtained results statistically processed. In order to assess the structure behavior, its resistance is compared with the effect of loads. A reliability index, necessary to quantify the structural safety, is then computed.

\subsubsection{Bayesian inference}

Bayesian methods can be used to incorporate external information into data analysis process, with the aim of reducing the statistical uncertainty $[11,12,14]$. This process starts with a given distribution, designated as prior, whose parameters may be chosen or estimated based on bibliography, experience or from experimental data. As more data is collected, Bayesian analysis is used to update the prior distribution into a posterior distribution. The Bayes theorem, which weights the prior information with evidence provided by new data, is the basic tool for the updating procedure.

Within the Bayesian approach, the choice of a prior distribution is considered as an extremely important step. The use of a non-informative prior is often useful when no prior information exists, but it is always necessary to check if the obtained posterior distribution is proper [30]. A common non-informative prior is the Jeffrey's prior. Although it is verified that traditional non-informative priors are improper, in most situations, computed posterior distributions are proper. When there is any information regarding the interest parameter, an informative 
prior may be used instead. This prior is not dominated by the likelihood and has an impact on the posterior distribution

Conjugacy corresponds to the property of a posterior distribution to follow the same parametric form of a prior distribution [30]. The use of conjugate families is convenient from a mathematical point of view, once the posterior distribution presents a known parametric form. In this case, obtained results are easy to understand and can be often represented in its analytical form. However, a more realistic prior may be used if there is information that contradicts the conjugate family.

The Bayesian inference procedure involves passing from a prior to a posterior distribution using the likelihood data function. Considering a Normal likelihood has the advantage of either conjugate or non-informative priors resulting in proper posteriors [30]. Within the Bayesian approach, interest parameters are assumed to follow certain probability distributions. Such distributions are defined by one or more unknown statistical parameters. These parameters are also considered to have given distributions. They are further updated given the data and will be respectively used to infer each interest parameter.

The aim of a Bayesian inference analysis is to obtain the posterior distribution. In several situations, it is enough to obtain point estimates that summarize the overall information (e.g. mean and variance parameters). Sometimes, this can be performed by using analytical closed form solutions, especially if prior distributions are properly chosen. One other alternative is to infer it from simulated distributions. According to Figure 1, a Bayesian inference algorithm is thus incorporated in the reliability assessment procedure [14].

\subsubsection{Reliability analysis}

Once the PDF of all variables is known and updated based on the experimental data, the simulation algorithm is used to generate values for those variables, compute the structural response and to evaluate each limit function $[1,2]$. From all these algorithms, the stratified sampling procedures, in particular the LHS, was commonly used due to its lower computational cost [4, 28, 29]. Accordingly, a LHS toolbox with an incorporated Iman and Conover [31] algorithm, is respectively used for generating the correlated random variables.

Structural safety is quantified through the comparison of resistance, $R$, and effect of loads, $S$, PDF [3]. In this situation, if $R$ and $S$ are independent random variables, the limit function $g(X)$ is given by $Z(R, S)=R-S[1,2]$. Accordingly, the failure probability, $p_{f}$, is expressed through equation (10), 
$p_{f}=P(Z(R, S) \leq 0) \Leftrightarrow P(R \leq S) \Leftrightarrow P(R-S \leq 0)$

The corresponding reliability index, $\beta$, is given by expression (11),

$\beta=-\phi^{-1}\left(p_{f}\right)$

being $\Phi^{-1}$ the inverse cumulative distribution function for a standard Normal PDF. The safety assessment procedure consists in a comparison between the computed and a target reliability index, $\beta_{\text {target }}$, given in codes or bibliography $[3,33]$.

\section{Reinforced concrete beams}

\subsection{Experimental tests}

Two reinforced concrete beams were tested, in laboratory, up to failure. These hyperstatic beams present a roller support in one end and a fixed support in the opposite side. Both beams present a rectangular cross section of $7.50 * 15.00 \mathrm{~cm}^{2}, b * h$, and a span, $L$, of $1.50 \mathrm{~m}$. Two equal loads are applied at middle span, being the distance between them of $50 \mathrm{~cm}$. Used materials are S500B reinforcing steel and C25/30 concrete [34]. Each beam has a superior and an inferior longitudinal reinforcing steel of, respectively, $2 \phi 8\left(A_{s}=1.005 \mathrm{~cm}^{2}\right)$ and $3 \phi 6\left(A_{s}=0.848\right.$ $\left.\mathrm{cm}^{2}\right)$, and a transversal reinforcing steel of $\phi 4 @ 0.03\left(A s_{w} / s=8.378 \mathrm{~cm}^{2} / \mathrm{m}\right)$, near supports, and of $\phi 4 @ 0.08$ $\left(A s_{w} / s=3.141 \mathrm{~cm}^{2} / \mathrm{m}\right)$, at middle span. An inferior, $c_{i n f}$, and superior, $c_{\text {sup }}$, concrete cover of $2.0 \mathrm{~cm}$ is considered. During the tests, the applied load, the middle span displacement and the roller support reaction, were continuously monitored. The test results show that the fixed support is not perfect, as rotation occurs, due to concrete accommodation. The failure load and maximum bending moment at fixed support are, respectively, of $30.52 \mathrm{kN}$ and $7.38 \mathrm{kN} . \mathrm{m}$, for beam 1 , and of $28.26 \mathrm{kN}$ and $6.43 \mathrm{kN} . \mathrm{m}$, for beam 2 .

\subsection{Numerical analysis}

The tested reinforced concrete beams were modelled using a NL FEM in ATENA ${ }^{\circledR}$ [35]. Concrete and steel are modeled according to stress-strain laws given in Figure $5[34,35]$. The stress-strain law considered for concrete is defined by the elasticity modulus, $E_{c}$, the compressive strain at compressive strength, $\varepsilon_{c}$, the compressive strength, $f_{c}$, the tensile strength, $f_{t}$, the critical displacement, $w_{d}$, and the fracture energy, $G_{f}$. The stress-strain law for reinforcing steel is described by the elasticity modulus, $E_{s}$, the yield strength, $\sigma_{y}$, the limit strain, $\varepsilon_{\text {lim }}$, and the 
ultimate strength, $\sigma_{u}$. Reinforcing steel is considered to be fully embedded in concrete. Vertical springs were considered at fixed support to simulate the concrete accommodation.

When performing several analysis of the same numerical model, as in probabilistic-based structural assessment, the computational cost issue becomes very important. In order to overcome it, the developed numerical model was simplified. Therefore, both finite element and load step numbers were optimized considering the model performance.

Figure 6a shows the numerical model deformation, crack pattern and horizontal strain of the modelled reinforced concrete beams. A bending failure mode, with concrete crushing and yielding of longitudinal reinforcing steel, is obtained. The collapse mechanism is characterized by two plastic hinges, one positioned at fixed support and the other close to the load on the opposite end of the beam. The numerical behavior of the analyzed reinforced concrete beams was similar to that obtained in experimental tests (Figure 6b).

\subsubsection{Sensitivity analysis}

Once the numerical model is developed, a sensitivity analysis was carried out to define the most important model parameters, and consequently reduce the computational cost. If the importance measure, see equation (1), is higher than $10 \%, b_{k, \text { lim }}$, the parameter will be considered as critical. This analysis was carried for both service load and failure load scenarios.

For service loads, see Figure $7 \mathrm{a}$, the most significant parameters are: concrete elasticity modulus, tensile strength, and fracture energy, inferior longitudinal reinforcing steel elasticity modulus and area, and beam width and height. Considering the analysis up to failure load (Figure $7 \mathrm{~b}$ ), it is verified that the concrete compressive strength, the inferior reinforcing steel yield strength and the inferior concrete cover are also significant.

Two further parameters, spring stiffness at fixed support, $k$, and load step in which the fixed support starts to work, were also considered in model identification. These parameters, although not considered in the sensitivity analysis, have a strong impact on the structural response. Accordingly, 9 and 12 parameters will be respectively considered in the analysis until service and failure loads.

\subsection{Model identification}

Once the numerical model and critical parameters are obtained, the following step consists in the application of the model identification procedure. The fitness function, equation (2), is the quadratic sum of two independent 
components, i.e., experimental and numerical applied load, $F$, and roller support reaction, $R$, respectively, for each registered middle span displacement, $\delta$, and bending moment at fixed support, $M$. A global optimization algorithm is then used to minimize this value.

An evolutionary strategies optimization algorithm in its plus version was used [17]. A parent population, $\mu$, and a parent for recombination, $\rho$, of 10 individuals, as well as an offspring population, $\lambda$, of 50 individuals are used. This algorithm stops as the fitness function convergence criterion or the maximum generation's number (1000) is attained. The generation gap, $n$, used for the fitness function criterion, equation (3), is established as $10 \%$ of the maximum generation's number. Therefore, the improvement on fitness value is evaluated from a gap of 100 generations. Once the algorithm stops, a population, constituted by different individuals, is obtained.

In case the convergence criterion is attained for any model, the process stops and the model is selected. Accordingly, if in the final population there are other models which accomplish this criterion, these models will be also extracted to a pool of models. In order to avoid falling into local minima, this algorithm is processed with different, randomly generated, starting points. Then, the best model selection is supported in a hybrid procedure which consists in evaluating the probability of each solution occurring based on previous knowledge followed by an expert judgment process. Those models for which the assessed parameter values are far from the initial ones, are placed in the lower part of the ranking. However, this does not mean that they are not chosen once the final decision can be made by the expert, who selects the model that provides more confidence.

\subsubsection{Convergence criterion}

In order to compute the threshold value, $\varepsilon$, for the fitness function convergence criterion, equation (3), it is first necessary to identify and quantify the different sources of errors (Table 1). When computing this value, the two independent components $i$ of the fitness function, equation (2), $\delta-F$ and $M-R$, are considered.

The following step consists in computing both experimental and numerical errors, for each fitness function component, through the law of propagation of uncertainty [22]. The standard error is calculated considering that a uniform PDF (type B) is respectively assigned to each source of error $j$ [22], obtained from Table 1. The partial derivative of the experimental and numerical results with respect to each standard error is unitary. Tables 2 and 3 show the experimental and numerical error computation for the analysis until failure load. 
Once the experimental and numerical errors are obtained, it will be possible to compute the fitness function uncertainty for each component $i$. In order to obtain this value, it is necessary to calculate the fitness function partial derivative in relation to the experimental and numerical results, by using expression (2). These values vary with the tested beam $k$, as they are proportional to maximum applied load and measured reaction. The law of propagation of uncertainty is then used to compute the fitness function uncertainty [22]. Table 4 present the fitness function uncertainty calculation for each tested beam.

The uncertainty in the fitness function improvement value and the corresponding threshold value computation is shown in Table 5. The fitness function criterion establishes that its improvement should be less than or equal to the computed threshold value. The threshold value for the analysis in service phase is obtained in a similar way, resulting in expression (12),

$\left\{\begin{array}{l}\text { Service } \rightarrow \varepsilon=4.17 \cdot 10^{-2}=4.17 \% \\ \text { Failure } \rightarrow \varepsilon=4.58 \cdot 10^{-2}=4.58 \%\end{array}\right.$

The threshold value can be understood as the model identification procedure precision. Accordingly, it is not meaningful to improve the fitness function to a value that is smaller than the precision.

\subsubsection{Results}

Obtained model identification results are given in Table 6. These results show that concrete and steel presents a lower strength than that initially predicted. However, a higher steel area is also obtained, balancing the $\sigma_{y} * A_{s}$ value used in NL FEA software [35]. Regarding geometric parameters, obtained value for concrete cover is close to that initially predicted, while lower values were identified for the beam dimensions.

In Figure 8a, the applied load is plotted against the middle span displacement, and in Figure $8 \mathrm{~b}$ the reaction at roller support is plotted against the bending moment at fixed support. From the analysis it is possible to conclude that the results from model identification until failure load are those that best fit the experimental curve.

Table 7 presents the fitness function values obtained by considering the initial values and those from model identification. It is verified that the fitness value obtained from the analysis until service load is always lower than that obtained from the same analysis until failure load, showing that the model is more accurate in service region. This is due to a higher number of critical parameters as well as of used points to compute the fitness 
function in this latter situation. However, for both situations, the developed procedure provided a considerable improvement in the performance estimates.

Table 8 provides both failure load and maximum bending moment at fixed support, computed for initial values and those from model identification in service phase and until failure load. Obtained error from model identification until failure load is lower than that given by initial values and by model identification in service phase. These results show that the analysis until failure load allows a significantly better prediction of the beam strength, as when applying the methodology in service phase, the model identification is performed for this region, being not possible to guarantee the curve fitting for failure region.

\subsection{Reliability assessment}

Once an updated numerical model is obtained from model identification, the next step consists in computing the resistance probability distribution of the assessed beam. During this analysis, both material (concrete and steel) and geometric properties (section dimensions and concrete cover) are considered as random variables [36, 37]. The mean value of all parameters is taken as the result of model identification, while distributions and coefficient of variation are defined based on bibliography [3, 4, 27], as presented in Table 9.

When complementary data is available, a Bayesian inference $[12,30]$ approach can be used to improve the reliability assessment procedure. The updating process uses data collected from material (concrete and steel) and geometric (concrete cover) tests [14]. An informative and a non-informative (Jeffrey's) prior were used in the Bayesian inference procedure with the aim of computing the posterior distribution. Of these two, the approach leading to a lower standard deviation of the posterior distribution will be used.

In this case, an Iman and Conover algorithm [31] is integrated in the LHS toolbox to generate samples of the correlated random variables [28, 29]. Used correlation values (Table 10) are obtained from bibliography [3, 4, 27]. A set of failure load values, $F_{R}$, are obtained from probabilistic analysis. These values are then statistically processed, and a PDF is fitted to the resistance samples. From this analysis it is verified that the Normal PDF presents an accurate fitting for all phases of the analysis.

\subsubsection{Characterization tests}

Complementary tests were developed at laboratory in order to characterize used concrete and steel as well as to control the concrete cover, as to provide means to quantify the accuracy of the proposed methodology. Obtained 
results are given at Table 6 . They confirm the lower concrete quality and the closeness of the concrete cover to the initial value. However, it is verified that the longitudinal reinforcing steel quality is higher than the expected. In fact, in terms of structural performance, the key parameter is $\sigma_{y} * A_{s}$, and the optimization algorithm provides several combinations of $\sigma_{y}$ and $A_{s}$ which gives very good results, being very difficult to distinguish between them and thus to identify the optimal combination.

\subsubsection{Bayesian inference}

The new information regarding material and geometric parameters collected through testing can be also used for model updating. In this situation, Bayesian inference is applied to update each model parameter distribution and consequently reduce the statistical uncertainty $[12,14,30]$. Regarding prior distributions, both Jeffrey's and conjugate priors were considered in this analysis. Used Bayesian inference methodology is exemplified here with an application to concrete compressive strength, for the case when both mean and variance are unknown.

In this situation, the following joint Jeffrey's improper prior is used, expression (13),

$p\left(\mu, \sigma^{2}\right) \propto 1 / \sigma^{2},-\infty<\mu<\infty, \sigma^{2}>0$

Using Bayes theorem [30], the posterior distribution given all observations $x_{i}$, is given by expression (14),

$p\left(\mu, \sigma^{2} \mid x\right) \propto\left(\frac{1}{\sigma^{2}}\right)^{1 / 2} \cdot \exp \left[-\frac{1}{2} \cdot\left(\frac{\mu-\bar{x}}{\sigma / \sqrt{n}}\right)^{2}\right] \cdot\left(\frac{1}{\sigma^{2}}\right)^{\frac{(n-1)+1}{2}} \cdot \exp \left[-\frac{1}{2} \cdot \frac{S}{2 \cdot \sigma^{2}}\right]$

where $S=\Sigma\left(x_{i}-\bar{x}\right)^{2}$ and $n$ the number of samples (in this case $n=5$ ). It is possible to conclude that the posterior distribution of $\mu$ conditional on $\sigma^{2}$ is given by a Normal PDF with mean $\bar{x}$ (in this case equal to 30.79 , Table 6) and variance $\sigma^{2} / n$, equation (15),

$\mu\left|\sigma^{2}, x \rightarrow N\left(\bar{x}, \sigma^{2} / n\right) \Rightarrow \mu\right| \sigma^{2}, X \rightarrow N\left(30.79, \sigma^{2} / 5\right)$

with the marginal posterior distribution of $1 / \sigma^{2}$, an inverse $\chi^{2}$ distribution, expression (16),

$\frac{(n-1) \cdot s^{2}}{\sigma^{2}} \rightarrow \chi_{n-1}^{2} \Rightarrow \frac{2.52}{\sigma^{2}} \rightarrow \chi_{n-1}^{2}$

where $s^{2}=\Sigma\left(x_{i}-\bar{x}\right)^{2} /(n-1)$ the sample variance (in this case, equals to 0.63). As $\sigma^{2}$ appears in conditional distribution $\mu \mid \sigma^{2}$, this means that $\mu$ and $\sigma^{2}$ are dependent. In this case the parameter distribution values can be obtained by simulation, through the application of expressions (15) and (16), or by analytical solutions [30]. 
In this case, the natural conjugate prior has the following form, equation (17),

$p\left(\mu, \sigma^{2}\right) \propto\left(\frac{n_{0}}{\sigma^{2}}\right)^{1 / 2} \cdot \exp \left[-\frac{n_{0}}{2 \cdot \sigma_{0}^{2}} \cdot\left(\mu-\mu_{0}\right)^{2}\right] \cdot\left(\frac{1}{\sigma^{2}}\right)^{v_{0} / 2+1} \cdot \exp \left[-\frac{S_{0}}{2 \cdot \sigma^{2}}\right]$

where $n_{0}$ is the initial sample size (in this case, the same weight is given to prior and likelihood data, with $n_{0}=$ 5), $S_{0}=\Sigma\left(x_{i}-\bar{x}\right)^{2}$ is the prior value for $S$, obtained from the prior standard deviation $\sigma_{0}$ (equals to 3.30 ), and $\mu_{0}$ the prior mean value (equals to 33.00, Table 6). The prior is thus a Normal-Gamma PDF or, in other words, the product of an inverted Gamma PDF, with argument $\sigma^{2}$, and $v_{0}\left(v_{0}=n_{0}-1\right)$ degrees of freedom, by a Normal PDF with argument $\mu$, being the variance proportional to $\sigma^{2}$. The prior distribution of $\mu$ conditional on $\sigma^{2}$ is thus a Normal PDF with prior mean $\mu_{0}$ and variance $\sigma^{2} / n_{0}\left(\sigma\left(\mu_{0}\right)^{2}\right)$, expression (18),

$\mu\left|\sigma^{2} \rightarrow N\left(\mu_{0}, \sigma^{2} / n_{0}\right) \Rightarrow \mu\right| \sigma^{2} \rightarrow N\left(33.00, \sigma^{2} / 5\right)$

being the prior distribution of $1 / \sigma^{2}$ a Gamma PDF with parameters $v_{0} / 2$ and $S_{0} / 2$, equation (19),

$1 / \sigma^{2} \rightarrow \operatorname{Gamma}\left(v_{0} / 2, S_{0} / 2\right) \Rightarrow 1 / \sigma^{2} \rightarrow \operatorname{Gamma}(2,21.78)$

The posterior distribution of $\mu$ conditional on $\sigma^{2}$ is a Normal PDF with mean $\mu_{l}$ and variance $\sigma^{2} / n_{l}\left(\sigma\left(\mu_{1}\right)^{2}\right)$, with $n_{l}=n_{0}+n$ the total samples size, expression (20),

$\mu\left|\sigma^{2} \rightarrow N\left(\mu_{1}, \sigma^{2} / n_{1}\right) \Rightarrow \mu\right| \sigma^{2} \rightarrow N\left(31.89, \sigma^{2} / 10\right)$

Being the marginal posterior distribution of $1 / \sigma^{2}$ a Gamma PDF, equation (21),

$1 / \sigma^{2} \mid x \rightarrow \operatorname{Gamma}\left(v_{1} / 2, S_{1} / 2\right) \Rightarrow 1 / \sigma^{2}, x \rightarrow \operatorname{Gamma}(4.5,29.15)$

with $v_{l}=v_{0}+n$ the posterior degrees of freedom. The posterior value for $S_{l}$ is thus obtained from expression (22),

$S_{1}=S_{0}+(n-1) \cdot s^{2}+\frac{n_{0} \cdot n}{n_{0}+n}\left(\bar{x}-\mu_{0}\right)^{2} \Rightarrow S_{1}=58.29$

Accordingly, the posterior sum of squares, $S_{l}$, combines the prior, $S_{0}$, and the sample sums, $s^{2}$, with the additional uncertainty given by the difference between the sample and the prior mean. It is possible to conclude that $\mu$ and $\sigma^{2}$ are dependent once $\sigma^{2}$ appears in conditional distribution $\mu \mid \sigma^{2}$. Therefore, the parameter distribution values can be obtained either by simulation, through expressions (20) and (21), or by analytical solutions [30]. Table 11 and Figure 9, respectively, present the posterior distributions parameter values and plot, obtained from the Bayesian inference analysis. 
In this case, as the Jeffrey's prior gives a lower standard deviation value for concrete compressive strength $(1.38<3.03)$, this distribution will be used in the reliability analysis. Table 9 presents the mean and standard deviation value for all model parameters, considering a Normal PDF [3]. The results from Bayesian inference analysis are respectively provided between brackets $[14,36]$. In this case, when sampling, the correlation coefficients provided in Table 10 are used.

In this situation, both materials (concrete and steel) and geometric (concrete cover) parameters were updated with data collected from characterization tests. In respect to concrete, the posterior mean values are close to the prior ones, with exception of the concrete fracture energy. The Bayesian inference diminished the standard deviation value for concrete compressive strength and tensile strength. In respect to reinforcing steel elasticity modulus and yield strength, obtained mean values are close to prior ones. Regarding the inferior concrete cover obtained mean value increased with Bayesian inference.

\subsubsection{Results}

In this case, a set of failure load values, $F_{R}$, is obtained from LHS sampling. A curve fitting procedure is then developed in order to determine the most suitable PDF. According to this process, the Normal type is considered to be the PDF that better represents the obtained results.

Obtained parameter values are given in Table 12. From the analysis of these results, it is possible to conclude that: (1) obtained mean and standard deviation with initial values and with values from model identification until failure load are higher than those obtained with values from model identification in service phase; (2) the Bayesian inference lead to an increase of the mean and a decrease of the CV. Figure 10 shows the resistance PDFs for these parameter values (mean and standard deviation). Obtained curves with initial values and with values from model identification until failure load are very close.

\subsection{Safety assessment of an existing reinforced concrete beam}

The proposed framework is now applied in a simple example of safety assessment. An existing reinforced concrete beam, with the same characteristics of previous assessed beam and located in a residential building floor is considered. This beam is subjected to a loading that presents the same configuration as that from laboratory test, Figure 6a. According to EN 1991-1-1 [38], and for residential areas (category A), each of the two imposed loads, $Q_{k}$, is defined by a Gumbel PDF, being the characteristic value set to the $95^{\text {th }}$ percentile for a 
reference period of 50 years. The imposed load magnitude is $3.00 \mathrm{kN}$, with a $\mathrm{CV}$ of $35 \%$, corresponding to a mean value of $1.80 \mathrm{kN}$ and a standard deviation of $0.63 \mathrm{kN} \mathrm{[39].}$

This load is then compared to the failure load, $F_{R}$, computed in section 3.4.3 and shown in Table 12. The resistance and load curve are then compared through the limit state function, $Z$, which is defined through equation (23),

$Z=R-S=F_{R}-F_{S}$

where $F_{S}$ is the applied load.

The probability of failure is computed by sampling this equation using LHS resulting in the values shown in Table 13 for all phases described in Table 12.

Through the analysis of Table 13, it is possible to conclude that obtained $\beta$-value considering the values from model identification until failure load is lower than the $\beta$-value obtained by considering the initial values. A diminishment on $\beta$-value is verified when considering the values from model identification in service phase. An increase on $\beta$-value is verified with Bayesian inference.

The developed framework considers real data when: (1) adjusting the model parameter values to collected data from real structure, through the model identification procedure; (2) updating the probabilistic model through a Bayesian inference procedure. For these reasons, the model identification until failure with Bayesian updating provides the most accurate results. In this example, the building is of class 2 (apartment building) and of class B (normal cost of safety measure), according to JCSS [3]. Therefore, a target reliability index, $\beta_{\text {target }}$, of 3.30 is recommended. This will allow to conclude that the assessed beam is safe.

\section{Conclusions}

This paper presents an innovative framework for probabilistic-based structural assessment of existing structures which explicitly considers different sources of uncertainty. This methodology is composed by two main steps. In the first step the numerical model is updated through a model identification procedure. In a second step, the updated deterministic model is converted into a probabilistic model, by considering randomness in model parameters, and a reliability assessment procedure is developed. Moreover, each model parameter PDF may be then updated with complementary data, through a Bayesian inference algorithm. This way, the statistical uncertainty is objectively addressed. The proposed framework presents a high computational cost. In order to 
overcome this, a previous sensitivity analysis, in which the most important parameters are selected, is recommended.

The developed framework is tested and validated with a set of reinforced concrete beams, which were loaded up to failure in laboratory. A NL FEM was respectively developed [35], being then performed a sensitivity analysis in order to identify the critical parameters. Some of these parameters were also characterized through laboratory tests. A model identification procedure was then executed in order to update the numerical model with measured data. A robust optimization algorithm, based in evolutionary strategies in its plus version, was then used [17]. Both modelling and measurement errors were considered in the optimization algorithm fitness function stopping criterion. This process was developed both in service and in failure region. A reliability assessment procedure was then executed. In order to do this, a PDF was respectively assigned to each critical parameter. Some of these distributions were updated through a Bayesian inference approach, with results from laboratory tests. An updated resistance PDF for applied load is then obtained from this procedure. This resistance model is then used in a safety assessment example.

The main conclusions from developed framework and its application are: (1) model identification until failure load gives very good results, as errors less than $10 \%$ are obtained; (2) model identification in service phase gives good results only for service region, being the obtained results for failure region less accurate. Complementary tests are thus recommended in this situation; (3) the most accurate models are those with values from model identification until failure load, being always recommended the development of a model identification procedure before the reliability assessment; (4) Bayesian inference increases the accuracy of probabilistic models by reducing the statistical uncertainty $[12,30]$. Therefore, this assessment procedure is always recommended when complementary data is available.

Although the case study presented consists in a set of new structures tested in laboratory conditions, the methodology proposed is better suited to the assessment of existing structures, for which limited information on materials and geometry exists and capacity to perform semi-destructive tests is limited. The results show that a significant improvement on the reliability estimates can be obtained when using the proposed combination of model identification and reliability analysis, allowing more informed decisions regarding repair and retrofit of existing structures. With this framework it will be possible to assess the structural behavior through a more robust and accurate way. 


\section{References}

[1] Melchers RE. Structural reliability analysis and prediction: John Wiley \& Sons; 1999.

[2] Nowak AS, Collins KR. Reliability of Structures. McGraw-Hill: Thomas Casson; 2000.

[3] JCSS JCoSS. Probabilistic Model Code, 12th Draft. JCSS - Joint Committee on Structural Safety; 2001.

[4] Henriques AAR. Aplicação de novos conceitos de segurança no dimensionamento do betão estrutural [Ph.D.

Dissertation]. Porto, Portugal: Universidade do Porto; 1998; (Portuguese).

[5] Enright MP, Frangopol DM. Reliability-based condition assessment of deteriorating concrete bridges considering load redistribution. Structural Safety. 1999;21:159-95;

[6] Faber MH, Val DV, Stewart MG. Proof load testing for bridge assessment and upgrading. Engineering Structures. 2000;22:1677-89;

[7] Faber MH. Reliability based assessment of existing structures. Progress in Structural Engineering and Materials. 2000;2:247-53;

[8] Enevoldsen I. Experience with probabilistic-based assessment of bridges. Structural Engineering International. 2001;11:251-60;

[9] Casas JR, Wisniewski D. Safety requirements and probabilistic models of resistance in the assessment of existing railway bridges. Structure and Infrastructure Engineering. 2011;9:529-45;

[10] Caspeele R, Taerwe L. Influence of concrete strength estimation on the structural safety assessment of existing structures. Construction and Building Materials. 2014;62:77-84;

[11] Strauss A, Frangopol DM, Kim S. Use of monitoring extreme data for the performance prediction of structures: Bayesian updating. Engineering Structures. 2008;30:3654-66;

[12] Jacinto LA. Avaliação da Segurança de Pontes Existentes - Abordagem Probabilística Bayesiana [Ph.D. Dissertation]. Lisboa, Portugal: Universidade Nova de Lisboa; 2011; (Portuguese).

[13] Bergmeister K, Novák D, Pukl R, Cervenka V. Structural assessment and reliability analysis for existing engineering structures, theoretical background. Structure and Infrastructure Engineering. 2009;5:267-75;

[14] Matos JC. Uncertainty evaluation of reinforced concrete and composite structures behavior [Ph.D. Dissertation]. Guimarães, Portugal: University of Minho; 2013; 
[15] Novák D, Pukl R, Strauss A. Reliability/risk assessment of concrete structures: Methodology, software and case study. First Middle East Conference on Smart Monitoring, Assessment and Rehabilitation of Civil Structures. Dubai, UAE2011. p. 1-9.

[16] Rücker W, Hille F, Rohrmann R. F08a - Guideline for the Assessment of Existing Structures. In: SAMCO, editor. Berlin, Germany: Federal Institute of Materials Research and Testing (BAM); 2006. p. 48.

[17] Beyer H-G, Schwefel H-P. Evolution strategies - A comprehensive introduction. Natural Computing. 2002;1:3-52;

[18] Robert-Nicoud Y, Raphael B, Smith IFC. System Identification through Model Composition and Stochastic Search. Journal of Computing in Civil Engineering. 2005;19:239-47;

[19] Ravindram S, Kripakaran P, Smith IFC. Evaluating reliability of multiple-model system identification. 14th EG-ICE workshop. Maribor, Slovenia2007.

[20] Goulet J-A, Michel C, Smith IFC. Hybrid probabilities and error-domain structural identification using ambient vibration monitoring. Mechanical Systems and Signal Processing. 2013;37:199-212;

[21] Goulet J-A, Smith IFC. Structural identification with systematic errors and unknown uncertainty dependencies. Computers \& Structures. 2013;128:251-8;

[22] JCGM. Evaluation of measurement data - Guide to the expression of uncertainty in measurement. JCGM (GUM 1995 with minor corrections), Technical Report: JCGM 100:2008; 2008. p. 132.

[23] Cabral P. Erros e incertezas nas medições. Relatório Técnico, IEP - Instituto Eletrónico Português: Laboratório de Metrologia e Ensaios e ISEP - Instituto Superior de Engenharia do Porto, Departamento de Física; 2004. p. 116.

[24] Goulet J-A, Kripakaran P, Smith IFC. Langesand Bridge in Lucerne, Results from Phase-I Static-Load Tests. Lausanne, Switzerland: EPFL - École Polytechnique et Fédérale de Lausanne; 2009. p. 37.

[25] Goulet J-A, Kripakaran P, Smith I. Multimodel Structural Performance Monitoring. Journal of Structural Engineering. 2010;136:1309-18;

[26] Goulet J-A, Kripakaran P, Smith IFC. Estimation of modelling errors in structural system identification. Proceedings of 4th International Conference on Structural Health Monitoring of Intelligent Infrastructure (SHMII-4). Zurich, Switzerland2009. p. 359.

[27] Santa U, Bergmeister K, Strauss A. Discussion of stochastic models in structural engineering. fib Symposium 2004. Avignon, France2004. 
[28] Olsson A, Sandberg G, Dahlblom O. On Latin hypercube sampling for structural reliability analysis. Structural Safety. 2003;25:47-68;

[29] Shields MD, Teferra K, Hapij A, Daddazio RP. Refined Stratified Sampling for efficient Monte Carlo based uncertainty quantification. Reliability Engineering \& System Safety. 2015;142:310-25;

[30] Bernardo JM, Smith AFM. Bayesian Theory. Chichestc, England: John Wiley \& Sons, Ltd; 2004.

[31] Iman RL, Conover WJ. A distribution-free approach to inducing rank correlation among input variables. Communications in Statistics - Simulation and Computation. 1982;11:311-34;

[32] Vořechovský M, Novák D. Statistical correlation in stratified sampling. Proceedings of 9th International Conference on Applications of Statistics and Probability in Civil Engineering-ICASP. San Francisco, USA: Millpress, Rotterdam; 2003.p. 119-24.

[33] Steenbergen RDJM, Sýkora M, Diamantidis D, Holický M, Vrouwenvelder T. Economic and human safety reliability levels for existing structures. Structural Concrete. 2015;16:323-32;

[34] EN 1992-1-1. Eurocode 2: Design of concrete structures - Part 1-1: General Rules and Rules for Buildings. Brussels: European Committee for Standardization CEN; 2004.

[35] Cervenka V, Jendele L, Cervenka J. ATENA® Program Documentation, Part 1: Theory. Prague, Czech Republic2009.

[36] Matos JC, Cruz PJS, Valente MIB, Neves LC. An advanced reliability procedure for lifetime assessment of Structures: Application to reinforced concrete beams. fib 2012 Symposium. Stockholm, Sweden2012.

[37] Matos JC, Valente MIB, Cruz PJS. Nonlinear probabilistic analysis of reinforced concrete structures. 34th IABSE Symposium - Large structures and infrastructures for environmentally constrained and urbanized areas. Venice, Italy2010.

[38] EN 1991-1-1. Eurocode 1: Eurocode 1: Actions on Structures: Part 1-1: Densities, self-weight and imposed loads. Brussels: European Committee for Standardization CEN; 2003.

[39] Gulvanessian H, Holicky M. Eurocodes: using reliability analysis to combine action effects. Proceedings of the Institution of Civil Engineers - Structures and Buildings. 2005;158:243-52; 


\section{List of Tables}

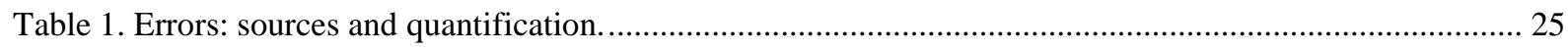

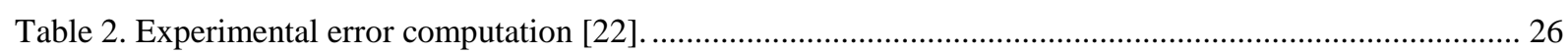

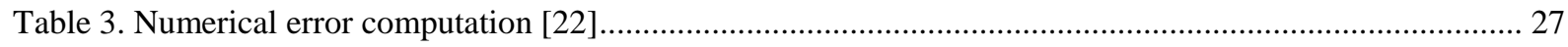

Table 4. Uncertainty calculation for each fitness function component [22] ....................................................28

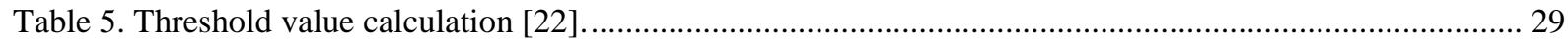

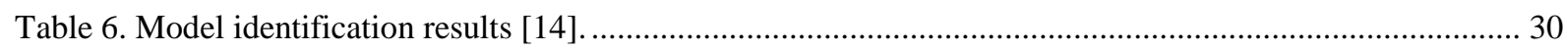

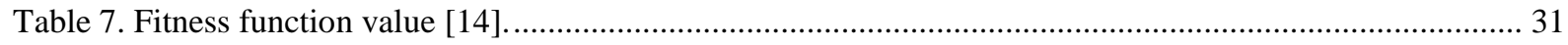

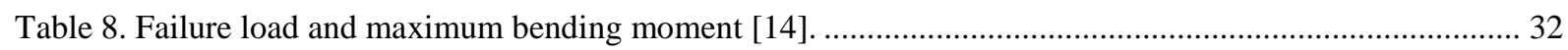

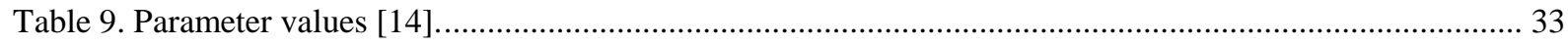

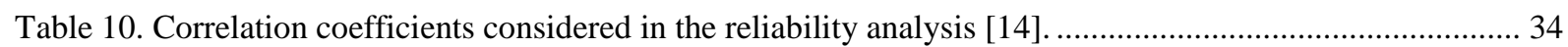

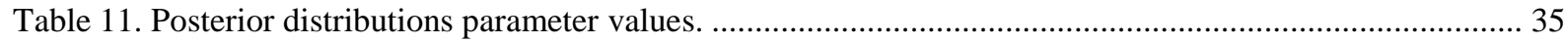

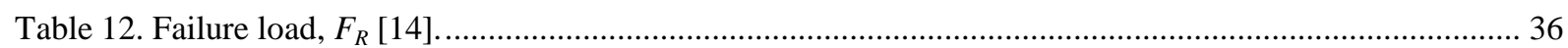

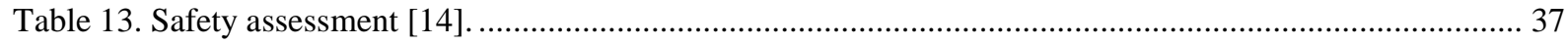

\section{List of Figures}

Figure 1. Probabilistic-based structural assessment algorithm [14] ........................................................ 38

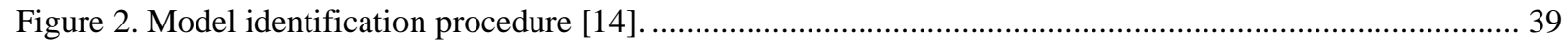

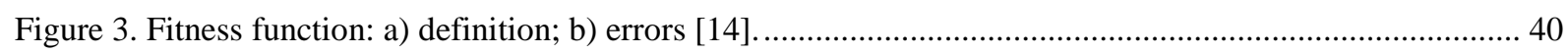

Figure 4. Modeling errors $u_{1}, u_{2}$ and $u_{3}$ (adapted from Ravindram et al. [19])..................................... 41

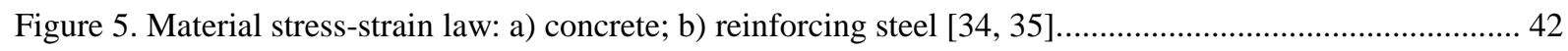

Figure 6. Collapse mechanism: a) numerical model; b) laboratory test [14]. ......................................... 43

Figure 7. Importance measure, $b_{k}$ : a) service load; b) failure load [14]. .............................................. 44

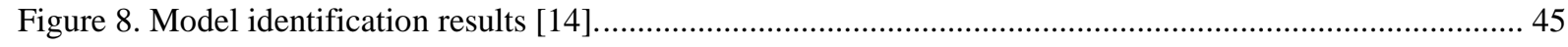

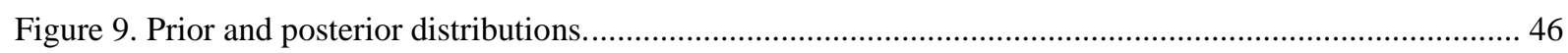

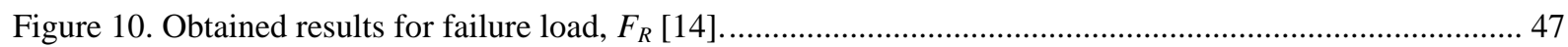


Table 1. Errors: sources and quantification.

\begin{tabular}{|c|c|c|c|}
\hline \multicolumn{2}{|c|}{ Sources } & Quantification method & Error $[\%]$ \\
\hline \multirow{5}{*}{ Experimental errors } & Sensor accuracy & $\begin{array}{c}\text { Manufacturer (includes } \\
\text { cable and acquisition } \\
\text { equipment losses) }\end{array}$ & $\begin{array}{c}0.10 \text { (displacement } \\
\text { transducer); } 0.10 \text { (load } \\
\text { cell) }\end{array}$ \\
\hline & Stability & $\begin{array}{l}\text { Static load test (no fatigue } \\
\text { problems detected) }\end{array}$ & $\rightarrow 0.00$ \\
\hline & Robustness & $\begin{array}{c}\text { Short term test } \\
\text { (environmental effects } \\
\text { neglected) }\end{array}$ & $\rightarrow 0.00$ \\
\hline & Load positioning & $\begin{array}{l}\text { Test assembly perfectly } \\
\text { controlled }\end{array}$ & $\rightarrow 0.00$ \\
\hline & Load intensity & $\begin{array}{c}\text { Manufacturer (includes } \\
\text { cable and acquisition } \\
\text { equipment losses) }\end{array}$ & 0.10 (load cell $)$ \\
\hline \multirow{4}{*}{ Numerical errors } & Finite element method & $\begin{array}{l}\text { Based on preliminary study } \\
\text { (by comparing to a more } \\
\text { refined mesh model) }\end{array}$ & $\begin{array}{c}11.42(\delta v s . F)^{*} ; 11.75 \\
(M v s . R)^{*}\end{array}$ \\
\hline & $\begin{array}{l}\text { Inaccurate } \\
\text { assumptions }\end{array}$ & $\begin{array}{c}\text { Based on preliminary study } \\
\text { (by comparing to a shorter } \\
\text { load step model) }\end{array}$ & $\begin{array}{c}5.91(\delta v s . F) * ; 6.16(M \\
v s . R) *\end{array}$ \\
\hline & Model exactitude & Model "as built" & $\rightarrow 0.00$ \\
\hline & Considered hypothesis & $\begin{array}{c}\text { Other hypothesis are } \\
\text { negligible }\end{array}$ & $\rightarrow 0.00$ \\
\hline
\end{tabular}

* Computed value for failure load [14]. 
Table 2. Experimental error computation [22].

\begin{tabular}{|c|c|c|c|c|c|}
\hline \multirow{2}{*}{ Component, $i$} & Error, $j$ & \multirow{2}{*}{ Type } & Standard error, $u_{\text {exp }, i j}$ & $\partial y^{e x p} / \partial u_{e x p, i j}$ & Experimental error, $u_{\text {exp }, i}$ \\
\hline & {$[\%]$} & & {$[-]$} & {$[\mathrm{kN}]$} & {$[\mathrm{kN}]$} \\
\hline$\delta-F$ & 0.10 & B & $(0.10 / 100) / \sqrt{ } 3=5.77 * 10^{-2}$ & 1 & $\begin{array}{c}\sqrt{\left(1^{2} *\left(5.77 * 10^{-2}\right)^{2}\right)=} \\
\quad=5.77 * 10^{-4}\end{array}$ \\
\hline$M-R$ & 0.10 & B & $(0.10 / 100) / \sqrt{ } 3=5.77 * 10^{-2}$ & 1 & $\begin{array}{c}\sqrt{\left(1^{2} *\left(5.77 * 10^{-2}\right)^{2}\right)=} \\
\quad=5.77 * 10^{-4}\end{array}$ \\
\hline
\end{tabular}


Table 3. Numerical error computation [22].

\begin{tabular}{|c|c|c|c|c|c|}
\hline \multirow{2}{*}{$\begin{array}{c}\text { Component, } \\
i \\
\end{array}$} & Error, $j$ & \multirow{2}{*}{ Type } & Standard error, $u_{n u m, i j}$ & $\partial y^{\text {num } / \partial u_{n u m, i j}}$ & Numerical error, $u_{n u m, i}$ \\
\hline & {$[\%]$} & & {$[-]$} & {$[\mathrm{kN}]$} & {$[\mathrm{kN}]$} \\
\hline \multirow{2}{*}{$\delta-F$} & 11.42 & B & $(11.42 / 100) / \sqrt{ } 3=6.59 * 10^{-2}$ & 1 & \multirow{2}{*}{$\begin{array}{c}\sqrt{ }\left(1^{2} *\left(6.59 * 10^{-2}\right)^{2}+\right. \\
\left.+1^{2} *\left(3.41 * 10^{2}\right)^{2}\right)= \\
\quad=7.42 * 10^{-2}\end{array}$} \\
\hline & 5.91 & B & $(5.91 / 100) / \sqrt{ } 3=3.41 * 10^{-2}$ & 1 & \\
\hline \multirow{2}{*}{$M-R$} & 11.75 & B & $(11.75 / 100) / \sqrt{ } 3=6.78 * 10^{-2}$ & 1 & \multirow{2}{*}{$\begin{array}{c}\sqrt{ }\left(1^{2} *\left(6.78 * 10^{-2}\right)^{2}+\right. \\
\left.+1^{2} *\left(3.56 * 10^{-2}\right)^{2}\right)= \\
\quad=7.66 * 10^{-2}\end{array}$} \\
\hline & 6.16 & B & $(6.16 / 100) / \sqrt{ } 3=3.56 * 10^{-2}$ & 1 & \\
\hline
\end{tabular}


Table 4. Uncertainty calculation for each fitness function component [22].

\begin{tabular}{|c|c|c|c|c|c|c|}
\hline \multirow{2}{*}{$\begin{array}{c}\text { Component, } \\
i\end{array}$} & \multirow{2}{*}{$\begin{array}{l}\text { Beam, } \\
\quad k\end{array}$} & $\partial f / \partial y^{\exp , i k}$ & $\partial f / \partial y^{n u m, i k}$ & Fitness uncertainty, $u_{f, i k}$ & $\partial f / \partial f_{i k}$ & $\begin{array}{l}\text { Fitness uncertainty, } \\
u_{f, i}\end{array}$ \\
\hline & & {$\left[\mathrm{kN}^{-1}\right]$} & {$\left[\mathrm{kN}^{-1}\right]$} & {$[-]$} & {$[-]$} & {$[-]$} \\
\hline \multirow{2}{*}{$\delta-F$} & $\begin{array}{c}\text { Beam } \\
1\end{array}$ & $3.30 * 10^{-2}$ & $3.30 * 10^{-2}$ & $\begin{array}{c}\sqrt{\left(\left(3.30 * 10^{-2}\right)^{2} *\left(5.77 * 10^{-4}\right)^{2}+\right.} \\
\left.+\left(3.30 * 10^{-2}\right)^{2} *\left(7.42 * 10^{-2}\right)^{2}\right)= \\
=2.43 * 10^{-3}\end{array}$ & 1 & \multirow{2}{*}{$\begin{array}{l}\sqrt{ }\left(1^{2} *\left(2.43 * 10^{-3}\right)^{2}+\right. \\
\left.+1^{2} *\left(2.63 * 10^{-3}\right)^{2}\right)= \\
\quad=3.58 * 10^{-2}\end{array}$} \\
\hline & $\begin{array}{c}\text { Beam } \\
2\end{array}$ & $3.60 * 10^{-2}$ & $3.60 * 10^{-2}$ & $\begin{array}{c}\sqrt{\left(\left(3.60 * 10^{-2}\right)^{2} *\left(5.77 * 10^{-4}\right)^{2}+\right.} \\
\left.+\left(3.60 * 10^{-2}\right)^{2} *\left(7.42 * 10^{-2}\right)^{2}\right)= \\
=2.63 * 10^{-3}\end{array}$ & 1 & \\
\hline \multirow{2}{*}{$M-R$} & $\begin{array}{c}\text { Beam } \\
1\end{array}$ & $1.36^{*} 10^{-1}$ & $1.36 * 10^{-1}$ & $\begin{array}{c}\sqrt{\left(\left(1.36 * 10^{-1}\right)^{2} *\left(5.77 * 10^{-4}\right)^{2}+\right.} \\
\left.+\left(1.36 * 10^{-1}\right)^{2} *\left(7.66 * 10^{-2}\right)^{2}\right)= \\
=1.04 * 10^{-2}\end{array}$ & 1 & \multirow{2}{*}{$\begin{array}{l}\sqrt{ }\left(1^{2} *\left(1.04 * 10^{-2}\right)^{2}+\right. \\
\left.+1^{2} *\left(1.19 * 10^{-2}\right)^{2}\right)= \\
\quad=1.58 * 10^{-2}\end{array}$} \\
\hline & $\begin{array}{c}\text { Beam } \\
2\end{array}$ & $1.56 * 10^{-1}$ & $1.56 * 10^{-1}$ & $\begin{array}{c}\sqrt{\left(\left(1.56 * 10^{-1}\right)^{2} *\left(5.77 * 10^{-4}\right)^{2}+\right.} \\
\left.+\left(1.56 * 10^{-1}\right)^{2} *\left(7.66 * 10^{-2}\right)^{2}\right)= \\
=1.19 * 10^{-2}\end{array}$ & 1 & \\
\hline
\end{tabular}


Table 5. Threshold value calculation [22].

\begin{tabular}{|c|c|c|c|c|c|c|c|c|}
\hline \multirow{2}{*}{$\begin{array}{c}\text { Component, } \\
i\end{array}$} & $\partial f / \partial f_{i}$ & $\begin{array}{c}\text { Fitness uncertainty, } \\
u_{f}\end{array}$ & $\partial \Delta f / \partial f_{i}$ & $\partial \Delta f / \partial f_{i+n}$ & $\begin{array}{c}\text { Improvement } \\
\text { uncertainty, } u_{\Delta f}\end{array}$ & $\begin{array}{l}\text { Coverage } \\
\text { factor, } k\end{array}$ & \multicolumn{2}{|c|}{$\begin{array}{l}\text { Threshold } \\
\text { value, } \varepsilon\end{array}$} \\
\hline & {$[-]$} & {$[-]$} & {$[-]$} & {$[-]$} & {$[-]$} & {$[-]$} & {$[-]$} & [\%] \\
\hline$\delta-F$ & 1 & \multirow{2}{*}{$\begin{array}{c}\sqrt{ }\left(1^{2} *\left(3.58 * 10^{-2}\right)^{2}+\right. \\
\left.+1^{2} *\left(1.58 * 10^{-2}\right)^{2}\right)= \\
\quad=1.62 * 10^{-2}\end{array}$} & \multirow[t]{2}{*}{1} & \multirow[t]{2}{*}{1} & \multirow{2}{*}{$\begin{array}{l}\sqrt{ }\left(1^{2} *\left(1.62 * 10^{-2}\right)^{2}+\right. \\
\left.+1^{2} *\left(1.62 * 10^{-2}\right)^{2}\right)= \\
\quad=2.29 * 10^{-2}\end{array}$} & \multirow[t]{2}{*}{2} & \multirow{2}{*}{$4.58 * 10^{-2}$} & \multirow{2}{*}{4.58} \\
\hline$M-R$ & 1 & & & & & & & \\
\hline
\end{tabular}


Table 6. Model identification results [14].

\begin{tabular}{|c|c|c|c|c|c|c|c|c|c|}
\hline \multirow{2}{*}{\multicolumn{5}{|c|}{ Numerical model }} & \multirow{2}{*}{ PDF } & \multirow{2}{*}{$\begin{array}{c}\text { Experimental } \\
\text { value }\end{array}$} & \multirow{2}{*}{$\begin{array}{l}\text { Initial } \\
\text { value }\end{array}$} & \multicolumn{2}{|c|}{ Model identification } \\
\hline & & & & & & & & Service & Failure \\
\hline \multirow{12}{*}{ 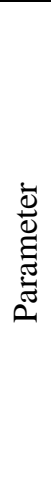 } & \multirow{7}{*}{ Material } & \multirow{4}{*}{ Concrete } & $E_{c}$ & {$[\mathrm{GPa}]$} & Normal & 28.01 & 31.00 & 30.34 & 29.07 \\
\hline & & & $f_{t}$ & {$[\mathrm{MPa}]$} & Normal & 2.67 & 2.60 & 2.45 & 2.63 \\
\hline & & & $f_{c}$ & [MPa] & Normal & 30.79 & 33.00 & 33.00 & 30.74 \\
\hline & & & $G_{f}$ & {$[\mathrm{~N} / \mathrm{m}]$} & Normal & 103.91 & 65.00 & 63.40 & 67.00 \\
\hline & & \multirow{3}{*}{$\begin{array}{c}\text { Inferior } \\
\text { longitudinal } \\
\text { reinforcing steel }\end{array}$} & $E_{s}$ & [GPa] & Normal & 205.31 & 200.00 & 244.58 & 180.96 \\
\hline & & & $\sigma_{y}$ & [MPa] & Normal & 582.94 & 560.00 & 560.00 & 548.28 \\
\hline & & & $A_{s}$ & {$\left[\mathrm{~cm}^{2}\right]$} & Normal & - & 0.85 & 1.02 & 0.89 \\
\hline & \multirow{3}{*}{\multicolumn{2}{|c|}{ Geometric }} & $c_{\text {inf }}$ & {$[\mathrm{cm}]$} & Normal & 2.30 & 2.00 & 2.00 & 2.04 \\
\hline & & & $b$ & {$[\mathrm{~cm}]$} & Normal & - & 7.50 & 7.04 & 7.15 \\
\hline & & & $h$ & {$[\mathrm{~cm}]$} & Normal & - & 15.00 & 12.16 & 13.59 \\
\hline & \multirow{2}{*}{\multicolumn{2}{|c|}{ Mechanic }} & $k$ & {$[\mathrm{kN} / \mathrm{m}]$} & Normal & - & 149.13 & 164.21 & 112.75 \\
\hline & & & step & {$[-]$} & Normal & - & 30 & 26 & 25 \\
\hline
\end{tabular}


Table 7. Fitness function value [14].

\begin{tabular}{|c|c|c|c|c|}
\hline \multirow{2}{*}{ Numerical model } & \multicolumn{3}{|c|}{ Fitness function } \\
\cline { 2 - 5 } & \multicolumn{2}{|c|}{ Service } & Failure \\
\cline { 2 - 5 } & Value [\%] & Improvement [\%] & Value [\%] & Improvement [\%] \\
\hline Initial values & 15.09 & - & 21.73 & - \\
\hline Model identification & 6.75 & 55.26 & 15.50 & 28.69 \\
\hline
\end{tabular}


Table 8. Failure load and maximum bending moment [14].

\begin{tabular}{|c|c|c|c|c|c|}
\hline \multirow{2}{*}{ Numerical model } & \multicolumn{2}{c|}{ Failure load } & \multicolumn{2}{c|}{ Maximum bending moment } \\
\cline { 3 - 6 } & Value [kN] & Error [\%] * & Value [kN.m] & Error [\%] $*$ \\
\hline \multicolumn{2}{|c|}{ Initial values } & 29.01 & 1.29 & 6.04 & 12.46 \\
\hline \multirow{2}{*}{ Model identification } & Service & 25.51 & 13.20 & 5.56 & 19.42 \\
\cline { 2 - 6 } & Failure & 29.17 & 0.75 & 6.26 & 9.28 \\
\hline
\end{tabular}

* Comparing with the real failure load and maximum bending moment. 
Table 9. Parameter values [14].

\begin{tabular}{|c|c|c|c|c|c|c|c|c|}
\hline \multirow{2}{*}{ Parameter } & \multirow{2}{*}{ PDF } & \multicolumn{2}{|c|}{ Initial value } & \multicolumn{2}{c|}{ Model identification (service) } & \multicolumn{2}{c|}{ Model identification (failure) } \\
\cline { 4 - 9 } & & $\mu$ & $\sigma$ & $\mu$ & $\sigma$ & $\mu$ & $\sigma$ \\
\hline$E_{c}$ & {$[\mathrm{GPa}]$} & Normal & $31.00(30.10)$ & $3.10(4.79)$ & $30.34(29.68)$ & $3.03(4.51)$ & $29.07(28.76)$ & $2.91(3.36)$ \\
\hline$f_{t}$ & {$[\mathrm{MPa}]$} & Normal & $2.60(2.62)$ & $0.52(0.31)$ & $2.45(2.68)$ & $0.49(0.34)$ & $2.63(2.64)$ & $0.53(0.31)$ \\
\hline$f_{c}$ & {$[\mathrm{MPa}]$} & Normal & $33.00(30.79)$ & $3.30(1.38)$ & $33.00(30.79)$ & $3.30(1.38)$ & $30.74(30.69)$ & $3.07(1.16)$ \\
\hline$G_{f}$ & {$[\mathrm{~N} / \mathrm{m}]$} & Normal & $65.00(104.61)$ & $6.50(15.83)$ & $63.41(104.61)$ & $6.34(15.83)$ & $66.95(104.61)$ & $6.70(15.83)$ \\
\hline$E_{s}$ & {$[\mathrm{GPa}]$} & Normal & $200.00(202.48)$ & $10.00(11.69)$ & $244.58(223.98)$ & $12.23(14.20)$ & $180.96(192.84)$ & $9.05(12.09)$ \\
\hline$\sigma_{y}$ & {$[\mathrm{MPa}]$} & Normal & $560.00(579.59)$ & $28.00(27.85)$ & $560.00(579.59)$ & $28.00(27.85)$ & $548.28(579.59)$ & $27.41(27.85)$ \\
\hline$A_{s}$ & {$\left[\mathrm{~cm}^{2}\right]$} & Normal & 0.85 & 0.02 & 1.02 & 0.02 & 0.89 & 0.02 \\
\hline$c_{\text {inf }}$ & {$[\mathrm{cm}]$} & Normal & $2.00(2.14)$ & $0.40(0.48)$ & $2.00(2.14)$ & $0.40(0.48)$ & $2.04(2.16)$ & $0.41(0.46)$ \\
\hline$b$ & {$[\mathrm{~cm}]$} & Normal & 7.50 & 0.75 & 7.04 & 0.70 & 7.15 & 0.72 \\
\hline$h$ & {$[\mathrm{~cm}]$} & Normal & 15.00 & 1.50 & 12.16 & 1.22 & 13.59 & 1.36 \\
\hline
\end{tabular}


Table 10. Correlation coefficients considered in the reliability analysis [14].

\begin{tabular}{|c|c|c|c|c|c|c|c|c|c|c|}
\hline & $E_{c}$ & $f_{t}$ & $f_{c}$ & $G_{f}$ & $E_{s}$ & $\sigma_{y}$ & $A_{s}$ & $c_{\text {inf }}$ & $b$ & $h$ \\
\hline$E_{c}$ & 1.00 & 0.70 & 0.90 & 0.50 & 0.00 & 0.00 & 0.00 & 0.00 & 0.00 & 0.00 \\
\hline$f_{t}$ & 0.70 & 1.00 & 0.80 & 0.90 & 0.00 & 0.00 & 0.00 & 0.00 & 0.00 & 0.00 \\
\hline$f_{c}$ & 0.90 & 0.80 & 1.00 & 0.60 & 0.00 & 0.00 & 0.00 & 0.00 & 0.00 & 0.00 \\
\hline$G_{f}$ & 0.50 & 0.90 & 0.60 & 1.00 & 0.00 & 0.00 & 0.00 & 0.00 & 0.00 & 0.00 \\
\hline$E_{s}$ & 0.00 & 0.00 & 0.00 & 0.00 & 1.00 & 0.80 & 0.50 & 0.00 & 0.00 & 0.00 \\
\hline$\sigma_{y}$ & 0.00 & 0.00 & 0.00 & 0.00 & 0.80 & 1.00 & 0.50 & 0.00 & 0.00 & 0.00 \\
\hline$A_{s}$ & 0.00 & 0.00 & 0.00 & 0.00 & 0.50 & 0.50 & 1.00 & 0.00 & 0.00 & 0.00 \\
\hline$c_{\text {inf }}$ & 0.00 & 0.00 & 0.00 & 0.00 & 0.00 & 0.00 & 0.00 & 1.00 & 0.10 & 0.60 \\
\hline$b$ & 0.00 & 0.00 & 0.00 & 0.00 & 0.00 & 0.00 & 0.00 & 0.10 & 1.00 & 0.10 \\
\hline$h$ & 0.00 & 0.00 & 0.00 & 0.00 & 0.00 & 0.00 & 0.00 & 0.60 & 0.10 & 1.00 \\
\hline
\end{tabular}


Table 11. Posterior distributions parameter values.

\begin{tabular}{|c|c|c|}
\hline Parameter & Jeffrey's & Conjugate \\
\hline$\mu_{0}$ & - & 33 \\
\hline$\sigma_{0}$ & - & 3.3 \\
\hline$\mu_{1}$ & 30.79 & 31.89 \\
\hline$\sigma\left(\mu_{l}\right)$ & 0.56 & 0.91 \\
\hline$\sigma_{l}$ & 1.22 & 2.79 \\
\hline$\sigma\left(\sigma_{l}\right)$ & 0.58 & 0.76 \\
\hline$\mu_{\text {pop }}$ & 30.79 & 31.89 \\
\hline$\sigma_{\text {pop }}$ & 1.38 & 3.03 \\
\hline
\end{tabular}


Table 12. Failure load, $F_{R}[14]$.

\begin{tabular}{|l|c|c|c|}
\hline Numerical model & & & \\
& PDF & $\mu[\mathrm{kN}]$ & $\sigma[\mathrm{kN}]$ \\
\hline Initial values & & & \\
\hline Initial values + Bayesian inference & Normal & 28.49 & 3.79 \\
\hline Model identification (service) & Normal & 31.69 & 4.19 \\
\hline Model identification (service) + Bayesian inference & Normal & 25.12 & 3.37 \\
\hline Model identification (failure) & Normal & 27.82 & 3.66 \\
\hline Model identification (failure) + Bayesian inference & Normal & 27.79 & 3.71 \\
\hline
\end{tabular}


Table 13. Safety assessment [14].

\begin{tabular}{|c|c|c|}
\hline Numerical model & $p_{f}$ & $\beta$ \\
\hline Initial values & $2.33 * 10^{-10}$ & 6.23 \\
\hline Initial values + Bayesian inference & $6.81 * 10^{-11}$ & 6.42 \\
\hline Model identification (service) & $1.12 * 10^{-9}$ & 5.98 \\
\hline Model identification (service) + Bayesian inference & $1.92 * 10^{-10}$ & 6.26 \\
\hline Model identification (failure) & $3.41 * 10^{-10}$ & 6.17 \\
\hline Model identification (failure) + Bayesian inference & $1.49 * 10^{-10}$ & 6.30 \\
\hline
\end{tabular}




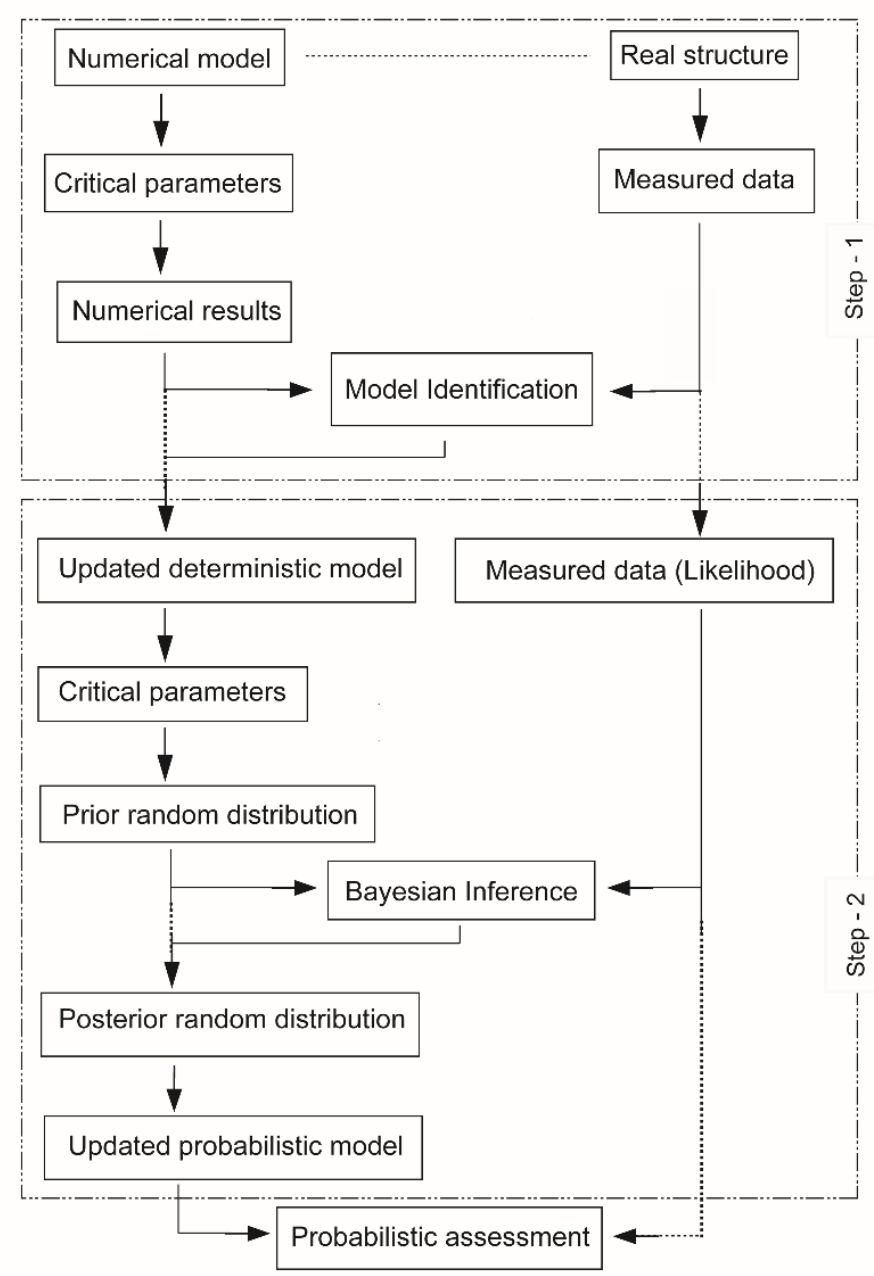

Figure 1. Probabilistic-based structural assessment algorithm [14]. 


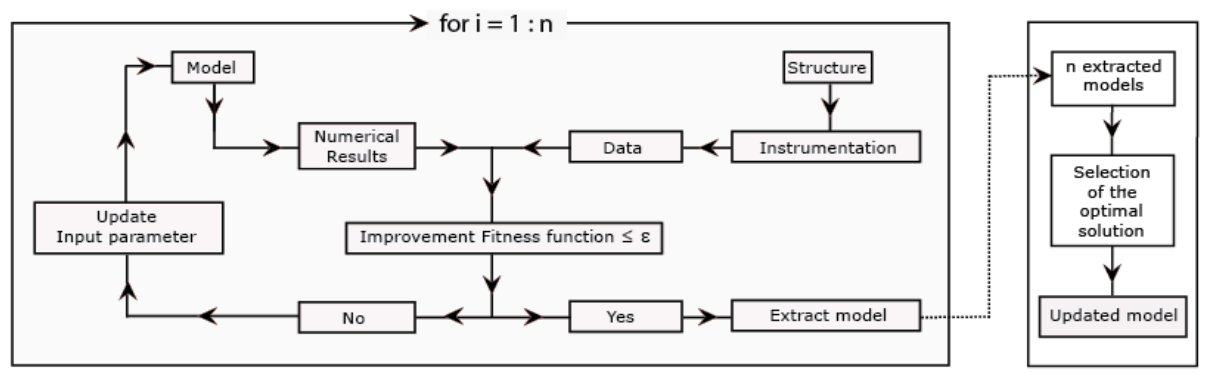

Figure 2. Model identification procedure [14]. 


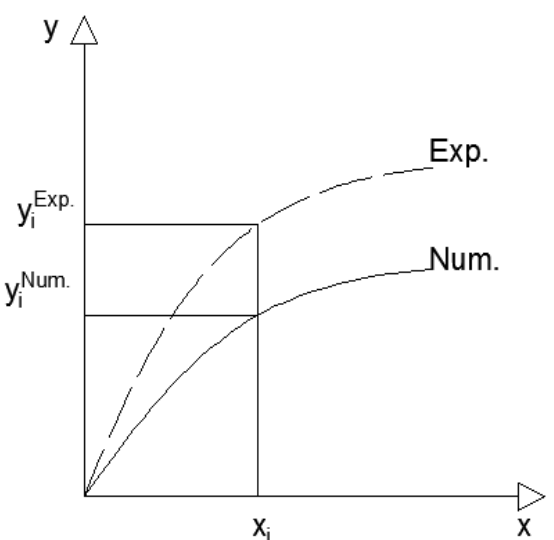

(a)

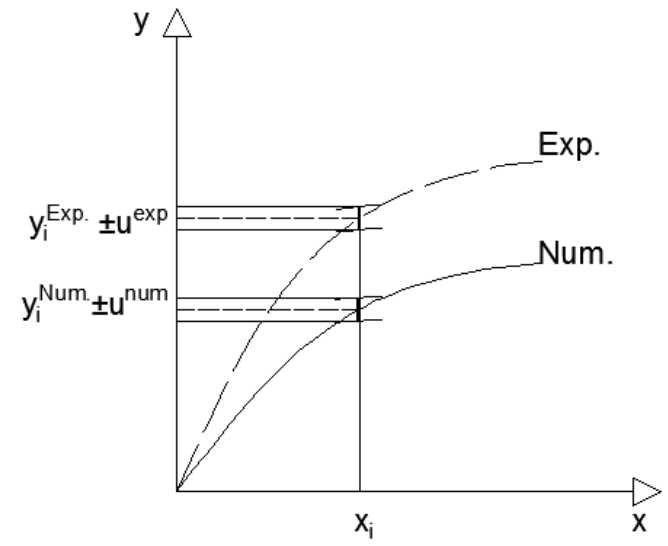

(b)

Figure 3. Fitness function: a) definition; b) errors [14]. 


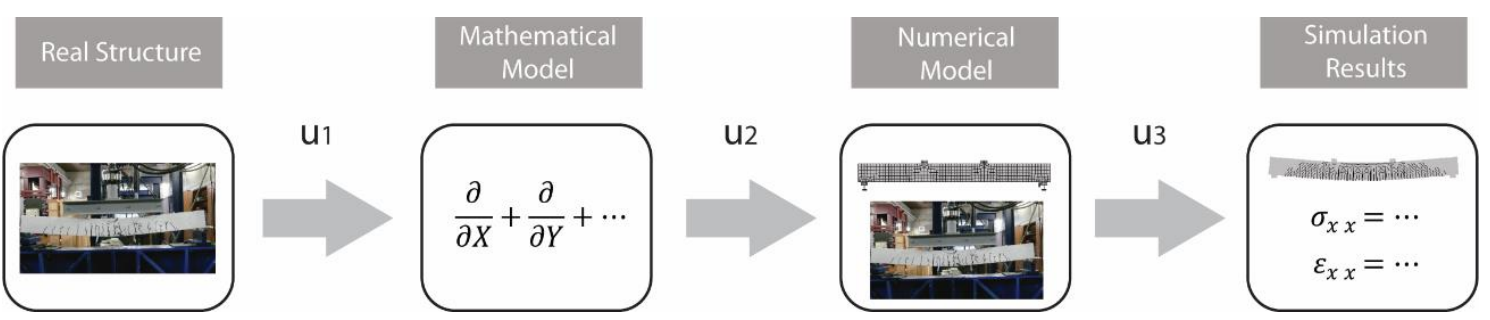

Figure 4. Modeling errors $u_{1}, u_{2}$ and $u_{3}$ (adapted from Ravindram et al. [19]). 


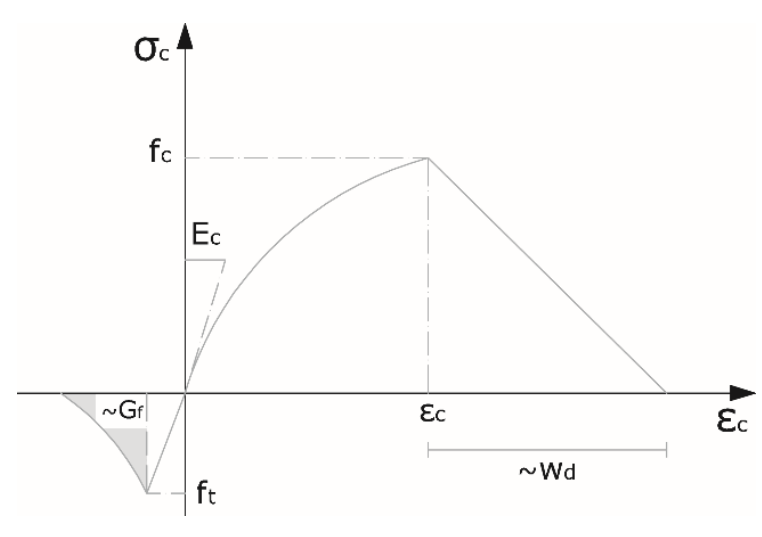

(a)

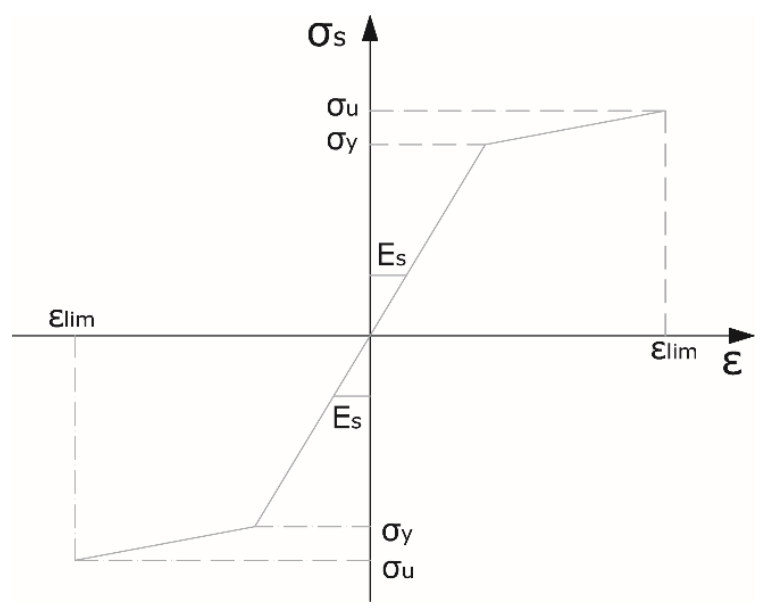

(b)

Figure 5. Material stress-strain law: a) concrete; b) reinforcing steel [34, 35]. 


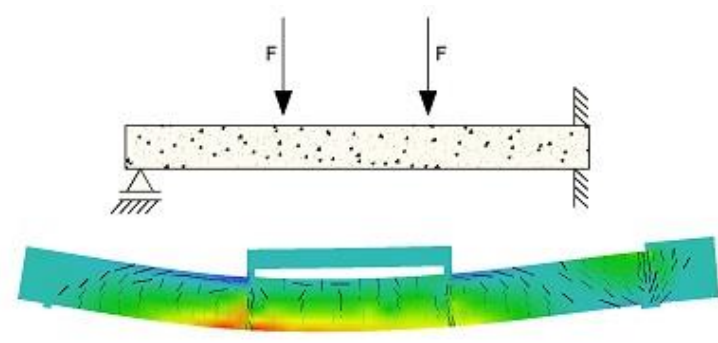

(a)

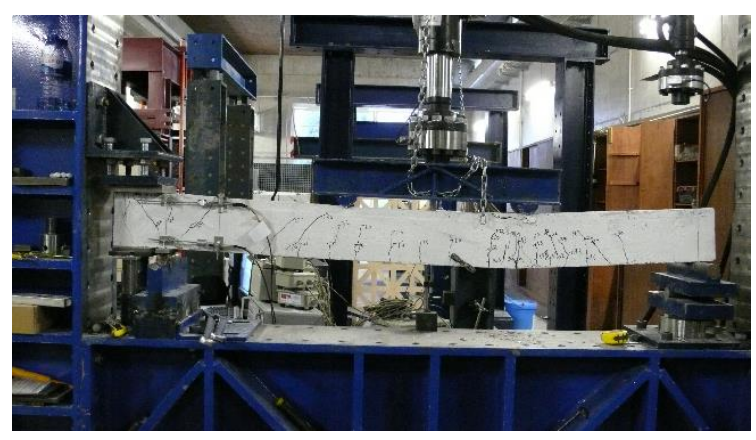

(b)

Figure 6. Collapse mechanism: a) numerical model; b) laboratory test [14]. 


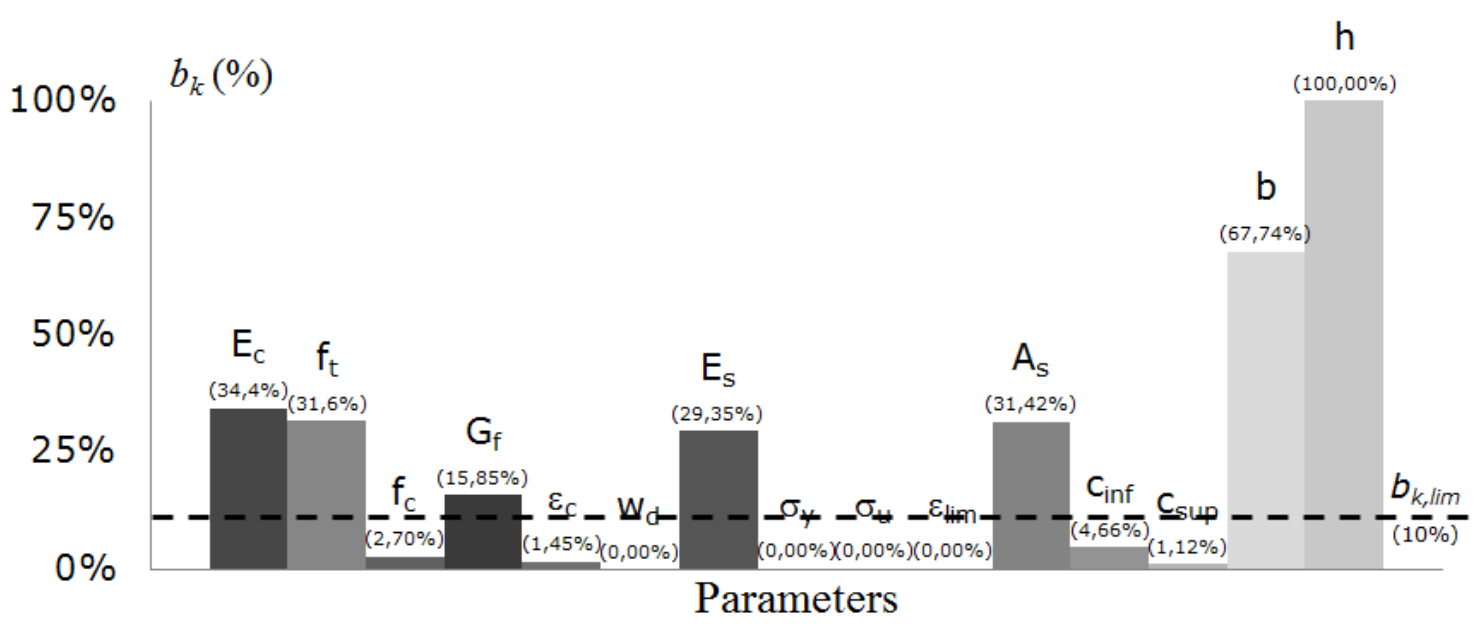

a)

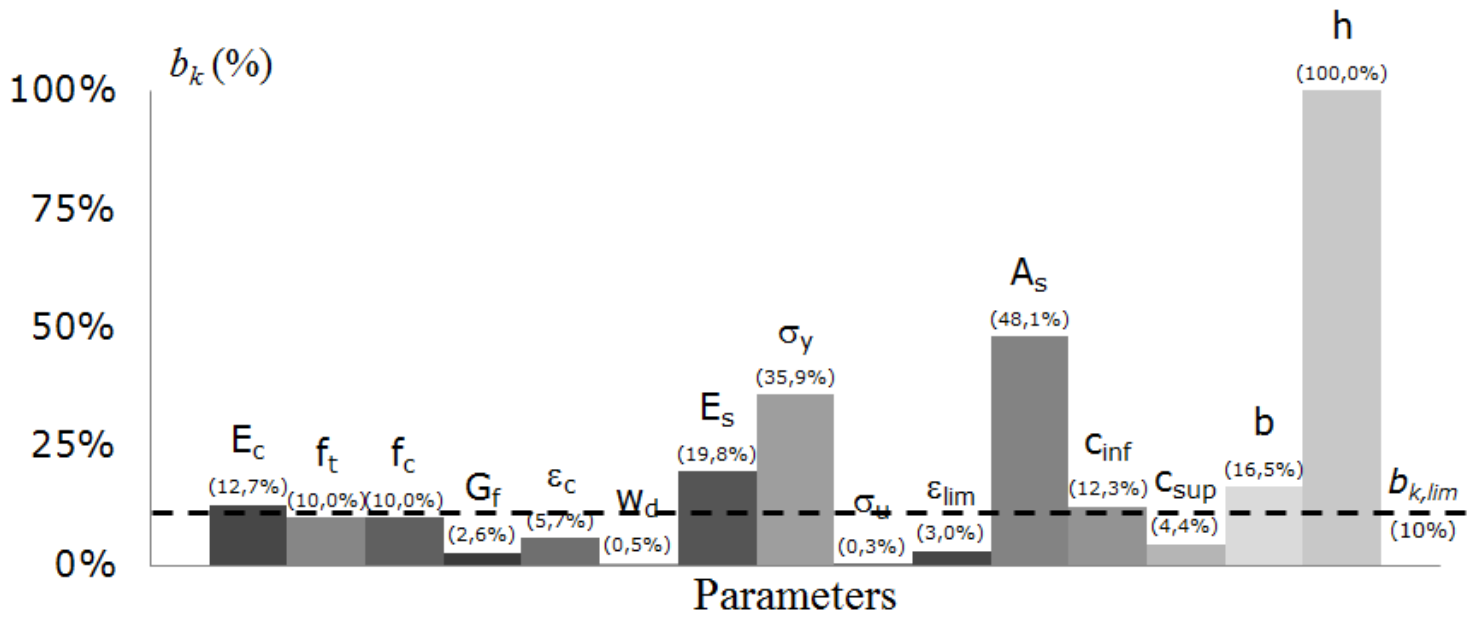

b)

Figure 7. Importance measure, $b_{k}$ : a) service load; b) failure load [14]. 


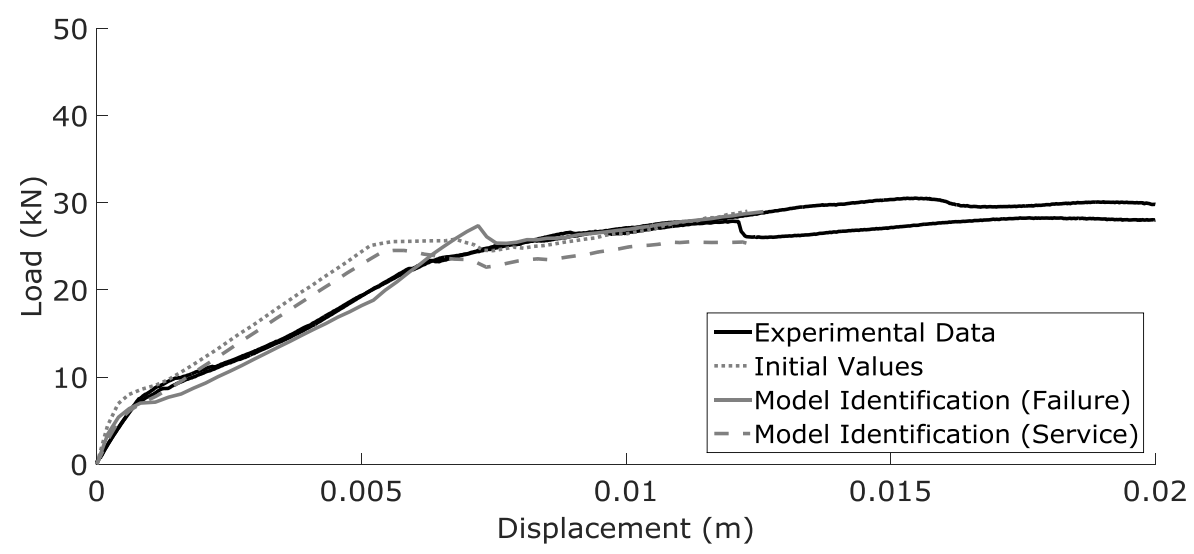

(a)

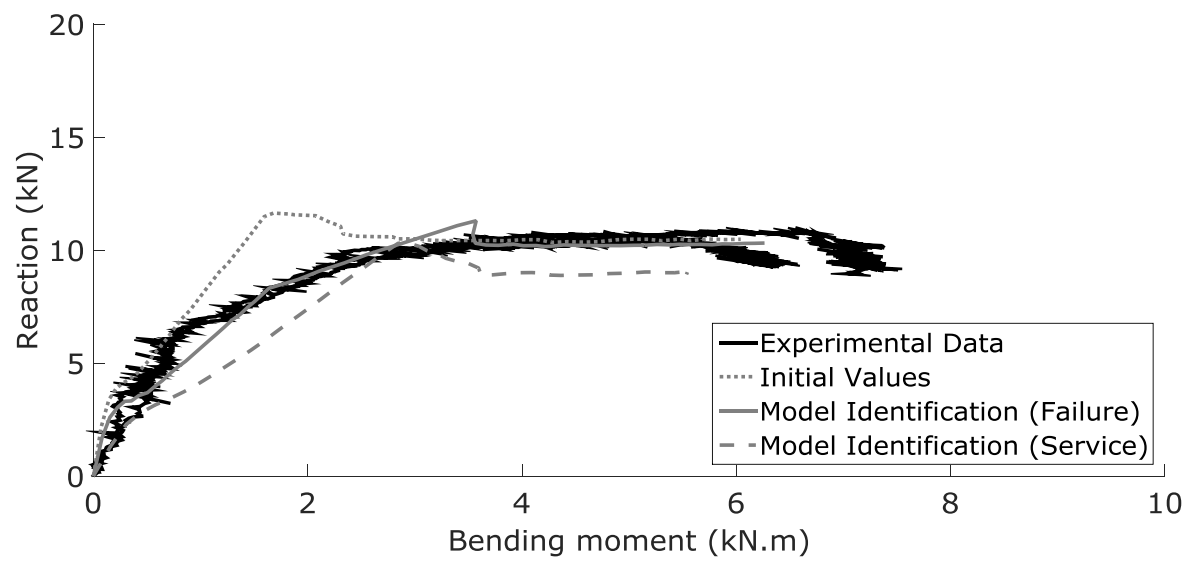

(b)

Figure 8. Model identification results [14]. 


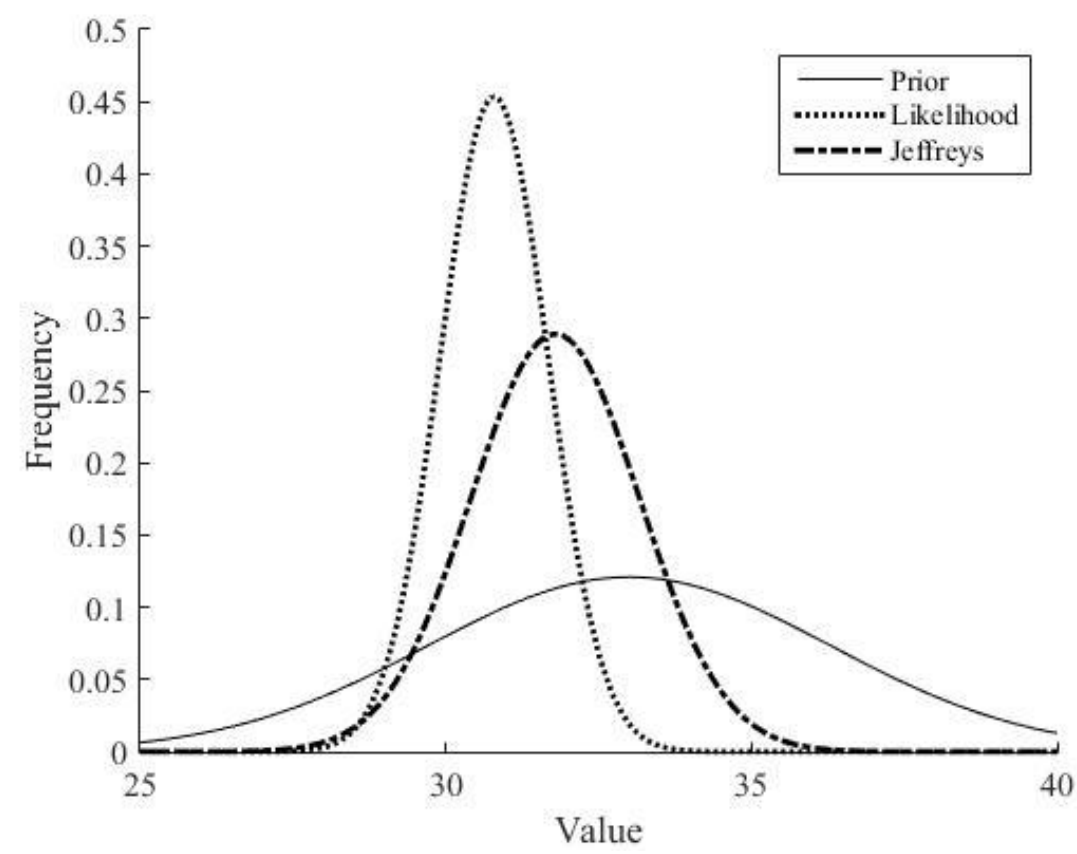

Figure 9. Prior and posterior distributions. 


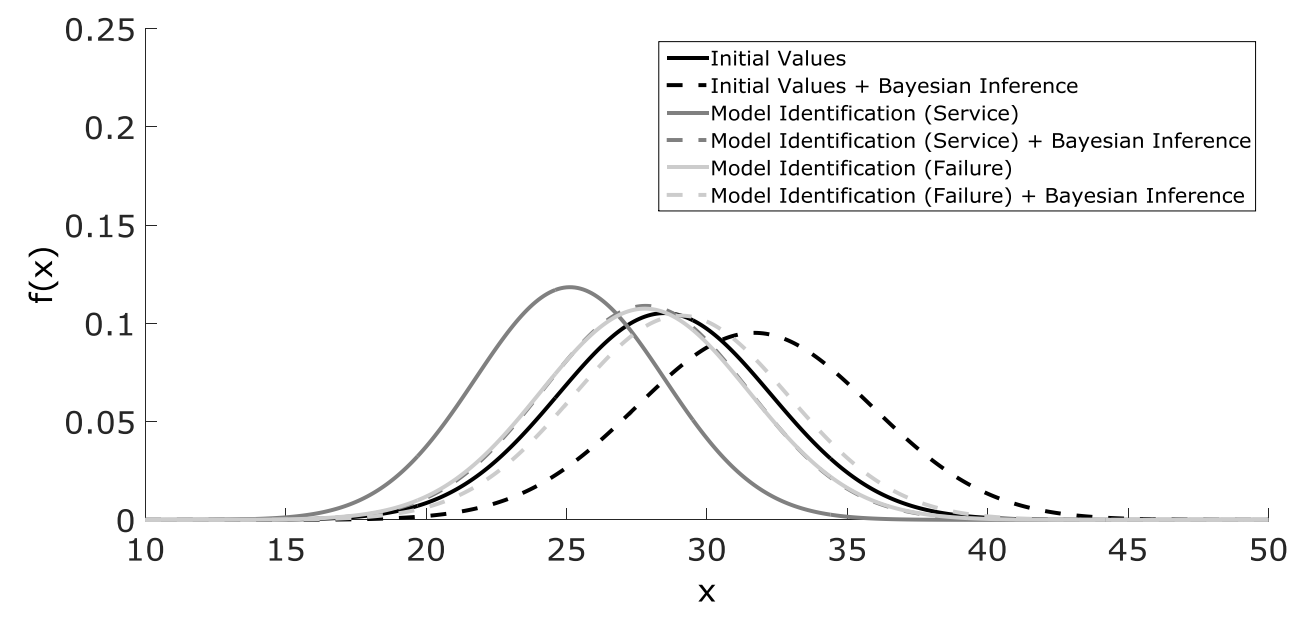

(a)

Figure 10. Obtained results for failure load, $F_{R}[14]$. 